\title{
Adaptive Thresholding for Sparse Covariance Matrix Estimation
}

\author{
Tony $\mathrm{Cai}^{1}$ and Weidong $\mathrm{Liu}^{1,2}$
}

\begin{abstract}
In this paper we consider estimation of sparse covariance matrices and propose a thresholding procedure which is adaptive to the variability of individual entries. The estimators are fully data driven and enjoy excellent performance both theoretically and numerically. It is shown that the estimators adaptively achieve the optimal rate of convergence over a large class of sparse covariance matrices under the spectral norm. In contrast, the commonly used universal thresholding estimators are shown to be sub-optimal over the same parameter spaces. Support recovery is also discussed. The adaptive thresholding estimators are easy to implement. Numerical performance of the estimators is studied using both simulated and real data. Simulation results show that the adaptive thresholding estimators uniformly outperform the universal thresholding estimators. The method is also illustrated in an analysis on a dataset from a small round blue-cell tumors microarray experiment. A supplement to this paper which contains additional technical proofs is available online.
\end{abstract}

Keywords: Adaptive thresholding, Frobenius norm, optimal rate of convergence, sparse covariance matrix, spectral norm, support recovery, universal thresholding.

\footnotetext{
${ }^{1}$ Department of Statistics, The Wharton School, University of Pennsylvania, Philadelphia, PA 19104, tcai@wharton.upenn.edu. The research was supported in part by NSF FRG Grant DMS-0854973.

${ }^{2}$ Department of Mathematics and Institute of Natural Sciences, Shanghai Jiao Tong University, China.
} 


\section{Introduction}

Let $\mathbf{X}=\left(X_{1}, \ldots, X_{p}\right)$ be a $p$-variate random vector with covariance matrix $\boldsymbol{\Sigma}_{0}$. Given an independent and identically distributed (i.i.d.) random sample $\left\{\mathbf{X}_{1}, \ldots, \mathbf{X}_{n}\right\}$ from the distribution of $\mathbf{X}$, we wish to estimate the covariance matrix $\boldsymbol{\Sigma}_{0}$ under the spectral norm. This covariance matrix estimation problem is of fundamental importance in multivariate analysis with a wide range of applications. The high dimensional setting, where the dimension $p$ can be much larger than the sample size $n$, is of particular current interest. In such a setting, conventional methods and results based on fixed $p$ and large $n$ are no longer applicable and new methods and theories are thus needed. In particular, the sample covariance matrix

$$
\boldsymbol{\Sigma}_{n}=\left(\hat{\sigma}_{i j}\right)_{p \times p}:=\frac{1}{n-1} \sum_{k=1}^{n}\left(\mathbf{X}_{k}-\overline{\mathbf{X}}\right)\left(\mathbf{X}_{k}-\overline{\mathbf{X}}\right)^{T}
$$

where $\overline{\mathbf{X}}=n^{-1} \sum_{k=1}^{n} \mathbf{X}_{k}$, performs poorly in this setting and structural assumptions are required in order to estimate the covariance matrix consistently.

In this paper we focus on estimating sparse covariance matrices. This problem has been considered in the literature. El Karoui (2008) and Bickel and Levina (2008) proposed thresholding of the sample covariance matrix $\Sigma_{n}$ and obtained rates of convergence for the thresholding estimators. Rothman, Levina and Zhu (2009) considered thresholding of the sample covariance matrix with more general thresholding functions. Cai and Zhou (2009 and 2010) established the minimax rates of convergence under the matrix $\ell_{1}$ norm and the spectral norm. Wang and Zou (2010) considered estimation of volatility matrices based on high-frequency financial data.

A common feature of the thresholding methods for sparse covariance matrix estimation proposed in the literature so far is that they all belong to the class of "universal thresholding rules". That is, a single threshold level is used to threshold all the entries of the sample covariance matrix. Universal thresholding rules were originally introduced by Donoho and 
Johnstone (1994 and 1998) for estimating sparse normal mean vectors in the context of wavelet function estimation. See also Antoniadis and Fan (2001). An important feature of problems considered there is that noise is homoscedastic. In such a setting, universal thresholding has demonstrated considerable success in nonparametric function estimation in terms of asymptotic optimality and computational simplicity.

In contrast to the standard homoscedastic nonparametric regression problems, sparse covariance matrix estimation is intrinsically a heteroscedastic problem in the sense that the entries of the sample covariance matrix could have a wide range of variability. Although some universal thresholding rules have been shown to enjoy desirable asymptotic properties, this is mainly due to the fact that the parameter space considered in the literature is relatively restrictive which forces the covariance matrix estimation problem to be an essentially homoscedastic problem.

To illustrate the point, it is helpful to consider an idealized model where one observes

$$
y_{i}=\mu_{i}+\gamma_{i} z_{i}, \quad z_{i} \stackrel{i i d}{\sim} N(0,1) \quad 1 \leq i \leq p
$$

and wishes to estimate the mean vector $\mu$ which is assumed to be sparse. If the noise levels $\gamma_{i}$ are bounded, say by $B$, then the universal thresholding rule $\hat{\mu}_{i}=y_{i} I\left(\left|y_{i}\right| \geq B \sqrt{2 \log p}\right)$ performs well asymptotically over a standard $\ell_{q}$ ball $\Theta_{q}\left(s_{0}\right)$ defined by

$$
\Theta_{q}\left(s_{0}\right)=\left\{\boldsymbol{\mu} \in \mathbb{R}^{p}: \sum_{j=1}^{p}\left|\mu_{j}\right|^{q} \leq s_{0}\right\} .
$$

In particular, $\Theta_{0}\left(s_{0}\right)$ is a set of sparse vectors with at most $s_{0}$ nonzero elements. Here the assumption that $\gamma_{i}$ are bounded by $B$ is crucial. The universal thresholding rule simply treats the heteroscedastic problem (2) as a homoscedastic one with all noise levels $\gamma_{i}=B$. It is intuitively clear that this method does not perform well when the range of $\gamma_{i}$ is large and it fails completely without the uniform boundedness assumption on the $\gamma_{i}$ 's.

For sparse covariance matrix estimation, the following uniformity class of sparse matri- 
ces was considered in Bickel and Levina (2008) and Rothman, Levina and Zhu (2009):

$$
\mathcal{U}_{q}:=\mathcal{U}_{q}\left(s_{0}(p)\right)=\left\{\boldsymbol{\Sigma}: \boldsymbol{\Sigma} \succ 0, \max _{i} \sigma_{i i} \leq K, \max _{i} \sum_{j=1}^{p}\left|\sigma_{i j}\right|^{q} \leq s_{0}(p)\right\}
$$

for some $0 \leq q<1$, where $\Sigma \succ 0$ means that $\Sigma$ is positive definite. Here each column of a covariance matrix in $\mathcal{U}_{q}\left(s_{0}(p)\right)$ is assumed to be in the $\ell_{q}$ ball $\Theta_{q}\left(s_{0}(p)\right)$. Define

$$
\theta_{i j}:=\operatorname{Var}\left(\left(X_{i}-\mu_{i}\right)\left(X_{j}-\mu_{j}\right)\right)
$$

where $\mu_{i}=\mathrm{E} X_{i}$. It is easy to see that in the Gaussian case, $\sigma_{i i} \sigma_{j j} \leq \theta_{i j} \leq 2 \sigma_{i i} \sigma_{j j}$. The condition $\max _{i} \sigma_{i i} \leq K$ for all $i$ ensures the variances of the entries of the sample covariance matrix to be uniformly bounded. Bickel and Levina (2008) proposed a universal thresholding estimator $\hat{\boldsymbol{\Sigma}}_{u}=\left(\hat{\sigma}_{i j}^{u}\right)$, where

$$
\hat{\sigma}_{i j}^{u}=\hat{\sigma}_{i j} I\left\{\hat{\sigma}_{i j} \geq \lambda_{n}\right\}
$$

and showed that with a proper choice of the threshold $\lambda_{n}$ the estimator $\hat{\boldsymbol{\Sigma}}_{u}$ achieves a desirable rate of convergence under the spectral norm. Rothman, Levina and Zhu (2009) considered a class of universal thresholding rules with more general thresholding functions than hard thresholding. Similar to the idealized model (21) discussed earlier, here a key assumption is that the variances $\sigma_{i i}$ are uniformly bounded by $K$ which is crucial to make the universal thresholding rules well behaved. A universal thresholding rule in this case essentially treats the problem as if all $\sigma_{i i}=K$ when selects the threshold $\lambda$.

For heteroscedastic problems such as sparse covariance matrix estimation, it is arguable more desirable to use thresholds that capture the variability of individual variables instead of using a universal upper bound. This is particularly true when the variances vary over a wide range or no obvious upper bound on the variances is known. A more natural and effective approach is to use thresholding rules with entry-dependent thresholds which automatically adapt to the variability of the individual entries of the sample covariance 
matrix. The main goal of the present paper is to develop such an adaptive thresholding estimator and study its properties.

In this paper we introduce an adaptive thresholding estimator $\hat{\Sigma}^{\star}=\left(\hat{\sigma}_{i j}^{\star}\right)_{p \times p}$ with

$$
\hat{\sigma}_{i j}^{\star}=s_{\lambda_{i j}}\left(\hat{\sigma}_{i j}\right)
$$

where $s_{\lambda}(z)$ is a general thresholding function similar to those used in Rothman, Levina and Zhu (2009) and will be specified later. The individual thresholds $\lambda_{i j}$ are fully datadriven and adapt to the variability of individual entries of the sample covariance matrix $\boldsymbol{\Sigma}_{n}$. It is shown that the adaptive thresholding estimator $\hat{\boldsymbol{\Sigma}}^{\star}$ enjoys excellent properties both asymptotically and numerically. In particular, we consider the performance of the estimator $\hat{\Sigma}^{\star}$ over a large class of sparse covariance matrices defined by

$$
\mathcal{U}_{q}^{\star}:=\mathcal{U}_{q}^{\star}\left(s_{0}(p)\right)=\left\{\Sigma: \Sigma \succ 0, \max _{i} \sum_{j=1}^{p}\left(\sigma_{i i} \sigma_{j j}\right)^{(1-q) / 2}\left|\sigma_{i j}\right|^{q} \leq s_{0}(p)\right\}
$$

for $0 \leq q<1$. In comparison to $\mathcal{U}_{q}\left(s_{0}(p)\right)$, the columns of a covariance matrix in $\mathcal{U}_{q}^{\star}$ are required to be in a weighted $\ell_{q}$ ball, instead of a standard $\ell_{q}$ ball, with the weight determined by the variance of the entries of the sample covariance matrix. A particular feature of $\mathcal{U}_{q}^{\star}$ is that it no longer requires the variances $\sigma_{i i}$ to be uniformly bounded and allows $\max _{i} \sigma_{i i} \rightarrow \infty$. Note that $\mathcal{U}_{q}\left(s_{0}(p)\right) \subseteq \mathcal{U}_{q}^{\star}\left(K^{1-q} s_{0}(p)\right)$, so the parameter space $\mathcal{U}_{q}^{\star}$ contains the uniformity class $\mathcal{U}_{q}$ as a subset. The parameter space $\mathcal{U}_{q}^{\star}$ can also be viewed as a weighted $\ell_{q}$ ball of correlation coefficients. See Section 3.1 for more discussions.

It will be shown in Section 3 that $\hat{\Sigma}^{\star}$ achieves the optimal rate of convergence

$$
s_{0}(p)\left(\frac{\log p}{n}\right)^{(1-q) / 2}
$$

over the parameter space $\mathcal{U}_{q}^{\star}\left(s_{0}(p)\right)$. In comparison, it is also shown that the best universal thresholding estimator can only attain the rate $s_{0}^{2-q}(p)\left(\frac{\log p}{n}\right)^{(1-q) / 2}$ over $\mathcal{U}_{q}^{\star}\left(s_{0}(p)\right)$, which is clearly sub-optimal when $s_{0}(p) \rightarrow \infty$ since $q<1$. 
The choice of the regularization parameters is important in any regularized estimation problem. The thresholds $\lambda_{i j}$ used in ([6]) are based on an estimator of the variance of the entries $\hat{\sigma}_{i j}$ of the sample covariance matrix. More specifically, $\lambda_{i j}$ are of the form

$$
\lambda_{i j}=\delta \sqrt{\frac{\hat{\theta}_{i j} \log p}{n}}
$$

where $\hat{\theta}_{i j}$ are estimates of $\theta_{i j}$ defined in (4) and $\delta$ is a tuning parameter. The value of $\delta$ can be taken as fixed at $\delta=2$, or it can be empirically chosen through cross validation. Theoretical properties of the resulting covariance matrix estimators using both methods are investigated. It is shown that the estimators attain the optimal rate of convergence under the spectral norm in both cases. In addition, support recovery of a sparse covariance matrix is also considered.

The adaptive thresholding estimators are easy to implement. Numerical performance of the estimators is investigated using both simulated and real data. Simulation results show that the adaptive thresholding estimators perform favorably in comparison to existing methods. In particular, they uniformly outperform the universal thresholding estimators in the simulation studies. The procedure is also applied to analyze a dataset from a small round blue-cell tumors microarray experiment (Khan et al., 2001).

The paper is organized as follows. Section 2 introduces the adaptive thresholding procedure for sparse covariance matrix estimation. Asymptotic properties are studied in Section 3. It is shown that the adaptive thresholding estimator is rate-optimal over $\mathcal{U}_{q}^{\star}$, while the best universal thresholding estimator is proved to be suboptimal. Section 4 discusses data-driven selection of the thresholds using cross validation and establish asymptotic optimality of the resulting estimator. Numerical performance of the adaptive thresholding estimators is investigated by simulations and by an application to a dataset from a small round blue-cell tumors microarray experiment in Section 5. Section 6 discusses methods based on the sample correlation matrix. The proofs are given in Section 7. 


\section{Adaptive thresholding for sparse covariance matrix}

In this section we introduce the adaptive thresholding method for estimating sparse covariance matrices. To motivate our estimator, consider again the sparse normal mean estimation problem (2). If the noise levels $\gamma_{i}^{\prime}$ 's are known or can be well estimated, a good estimator of the mean vector is the hard thresholding estimator $\hat{\mu}_{i}=y_{i} I\left\{\left|y_{i}\right| \geq \gamma_{i} \sqrt{2 \log p}\right\}$ or some generalized thresholding estimator with the same thresholds $\gamma_{i} \sqrt{2 \log p}$.

Similarly, for sparse covariance matrix estimation, a more effective thresholding rule than universal thresholding is the one which adapts to the variability of the individual entries of the sample covariance matrix. Define $\theta_{i j}$ as in (4). Then, roughly speaking, estimation of a sparse covariance matrix is similar to the mean vector estimation problem based on the observations

$$
\frac{1}{n} \sum_{k=1}^{n}\left(X_{k i}-\mu_{i}\right)\left(X_{k j}-\mu_{j}\right)=\sigma_{i j}+\sqrt{\frac{\theta_{i j}}{n}} z_{i j}, \quad 1 \leq i, j \leq p
$$

with $z_{i j}$ being asymptotically standard normal. This analogy provides a good motivation for our adaptive thresholding procedure. If the $\theta_{i j}$ were known, a natural thresholding estimator would be $\left(\hat{\sigma}_{i j}^{o}\right)_{p \times p}$ with

$$
\hat{\sigma}_{i j}^{o}=s_{\lambda_{i j}^{o}}\left(\hat{\sigma}_{i j}\right) \quad \text { with } \lambda_{i j}^{o}=2 \sqrt{\frac{\theta_{i j} \log p}{n}},
$$

where $s_{\lambda}(z)$ is a thresholding function. Comparing to the universal thresholding rule in Bickel and Levina (2008), the variance factors $\theta_{i j}$ in the thresholds make the thresholding rule entry-dependent and leads to a more flexible estimator. In practice, $\theta_{i j}$ are typically unknown, but can be well estimated. We propose the following estimator of $\theta_{i j}$ :

$$
\hat{\theta}_{i j}=\frac{1}{n} \sum_{k=1}^{n}\left[\left(X_{k i}-\bar{X}^{i}\right)\left(X_{k j}-\bar{X}^{j}\right)-\hat{\sigma}_{i j}\right]^{2}, \quad \bar{X}^{i}=n^{-1} \sum_{k=1}^{n} X_{k i} .
$$

This leads to our adaptive thresholding estimator of the covariance matrix $\Sigma_{0}$,

$$
\hat{\Sigma}^{\star}(\delta)=\left(\hat{\sigma}_{i j}^{\star}\right)_{p \times p} \quad \text { with } \quad \hat{\sigma}_{i j}^{\star}=s_{\lambda_{i j}}\left(\hat{\sigma}_{i j}\right)
$$


where

$$
\lambda_{i j}:=\lambda_{i j}(\delta)=\delta \sqrt{\frac{\hat{\theta}_{i j} \log p}{n}} .
$$

Here $\delta>0$ is a regularization parameter. It can be fixed at $\delta=2$ or can be chosen through cross validation. Good choices of $\delta$ will not affect the rate of convergence, but will affect the numerical performance of the resulting estimators. Selection of $\delta$ is thus of practical importance and we will discuss it further later.

The analogy between the sparse covariance estimation problem and the idealized mean estimation problem (9) gives a good motivation for the adaptive thresholding estimator defined in (11) and (12), but of course the matrix estimation problem is not exactly equivalent to the mean estimation problem (9) as noise is not exactly normal or iid and the loss is the spectral norm, not a vector norm or the Frobenius norm. We shall make our technical analysis precise in Sections 3 and 7.

In the present paper, we consider simultaneously a class of thresholding functions $s_{\lambda}(z)$ that satisfy the following conditions:

(i). $\left|s_{\lambda}(z)\right| \leq c|y|$ for all $z, y$ satisfy $|z-y| \leq \lambda$ and some $c>0$;

(ii). $s_{\lambda}(z)=0$ for $|z| \leq \lambda$;

(iii). $\left|s_{\lambda}(z)-z\right| \leq \lambda$, for all $z \in \mathbb{R}$.

These three conditions are satisfied, for example, by the soft thresholding rule $s_{\lambda}(z)=$ $\operatorname{sgn}(z)(z-\lambda)_{+}$and the adaptive lasso rule $s_{\lambda}(z)=z\left(1-|\lambda / z|^{\eta}\right)_{+}$with $\eta \geq 1$, as called in Rothman, Levina and Zhu (2009). We shall present a unified analysis of the adaptive thresholding estimators with the thresholding function $s_{\lambda}(z)$ satisfying the above three conditions. It should be noted that Condition (i) excludes the hard thresholding rule. However, all of the theoretical results in this paper hold for the hard thresholding estimator 
under similar conditions. Here Condition (i) is in place only to make the technical analysis in Section 7 work in a unified way for the class of thresholding rules. The results for the hard thresholding rule require slightly different proofs.

Rothman, Levina and Zhu (2009) proposed generalized universal thresholding estimators

$$
\hat{\mathbf{\Sigma}}_{g}=\left(\hat{\sigma}_{i j}^{g}\right)_{p \times p}, \text { where } \hat{\sigma}_{i j}^{g}=\bar{s}_{\lambda_{n}}\left(\hat{\sigma}_{i j}\right)
$$

and $\bar{s}_{\lambda}(z)$ satisfies (ii), (iii) and $\left|\bar{s}_{\lambda}(z)\right| \leq|z|$, which is slightly weaker than (i). Similar general universal thresholding rules were introduced and studied by Antoniadis and Fan (2001) in the context of wavelet function estimation. We should note that the generalized universal thresholding estimators $\hat{\boldsymbol{\Sigma}}_{g}$ suffer the same shortcomings as those of $\hat{\boldsymbol{\Sigma}}_{u}$, and like $\hat{\Sigma}_{u}$ they are sub-optimal over the class $\mathcal{U}_{q}^{\star}$.

\section{Theoretical properties of adaptive thresholding}

We now consider the asymptotic properties of the adaptive thresholding estimator $\hat{\Sigma}^{\star}(\delta)$ defined in (11) and (12). It is shown that the estimator $\hat{\boldsymbol{\Sigma}}^{\star}(\delta)$ adaptively attains the optimal rate of convergence over the collection of parameter spaces $\mathcal{U}_{q}^{\star}\left(s_{0}(p)\right)$.

We begin with some notation. Define the standardized variables

$$
Y_{i}=\left(X_{i}-\mu_{i}\right) /\left(\operatorname{Var}\left(X_{i}\right)\right)^{1 / 2}
$$

where $\mu_{i}=\mathrm{E} X_{i}$, and let $\mathbf{Y}=\left(Y_{1}, \ldots, Y_{p}\right)$. Throughout the paper, denote $|\mathbf{a}|_{2}=\sqrt{\sum_{j=1}^{p} a_{j}^{2}}$ for the usual Euclidean norm of a vector $\mathbf{a}=\left(a_{1}, \ldots, a_{p}\right)^{T} \in \mathbb{R}^{p}$. For a matrix $\boldsymbol{A}=\left(a_{i j}\right) \in$ $\mathbb{R}^{p \times q}$, define the spectral norm $\|\boldsymbol{A}\|_{2}=\sup _{|\mathbf{x}|_{2} \leq 1}|\boldsymbol{A x}|_{2}$, the matrix $\ell_{1}$ norm $\|\boldsymbol{A}\|_{L_{1}}=$ $\max _{1 \leq j \leq q} \sum_{i=1}^{p}\left|a_{i, j}\right|$, and the Frobenius norm $\|\boldsymbol{A}\|_{F}=\sqrt{\sum_{i, j} a_{i j}^{2}}$. For two sequences of real numbers $\left\{a_{n}\right\}$ and $\left\{b_{n}\right\}$, write $a_{n}=O\left(b_{n}\right)$ if there exists a constant $C$ such that $\left|a_{n}\right| \leq C\left|b_{n}\right|$ holds for all sufficiently large $n$, and write $a_{n}=o\left(b_{n}\right)$ if $\lim _{n \rightarrow \infty} a_{n} / b_{n}=0$. 


\subsection{Rate of convergence}

It is conventional in the covariance matrix estimation literature to divide the technical analysis into two cases according the the moment conditions on $\mathbf{X}$.

(C1). (Exponential-type tails) Suppose that $\log p=o\left(n^{1 / 3}\right)$ and there exists some $\eta>0$ such that

$$
\operatorname{E} \exp \left(t Y_{i}^{2}\right) \leq K_{1}<\infty \quad \text { for all }|t| \leq \eta \text { and } i
$$

Furthermore, we assume that for some $\tau_{0}>0$,

$$
\min _{i j} \operatorname{Var}\left(Y_{i} Y_{j}\right) \geq \tau_{0}
$$

(C2). (Polynomial-type tails) Suppose that for some $\gamma, c_{1}>0, p \leq c_{1} n^{\gamma}$, and for some $\epsilon>0$

$$
\mathrm{E}\left|Y_{i}\right|^{4 \gamma+4+\epsilon} \leq K_{1} \text { for all } i \text {. }
$$

Furthermore, we assume that (14) holds.

Remark 1 Note that (C1) holds with $\tau_{0}=1$ in the Gaussian case where $\mathbf{X} \sim N\left(\boldsymbol{\mu}, \boldsymbol{\Sigma}_{0}\right)$. To this end, let $\rho_{i j}$ be the correlation coefficient of $Y_{i}$ and $Y_{j}$. We can then write $Y_{i}=\rho_{i j} Y_{j}+$ $\sqrt{1-\rho_{i j}^{2}} Y$, where $Y \sim N(0,1)$ is independent of $Y_{j}$. So we have $\operatorname{Var}\left(Y_{i} Y_{j}\right)=1+\rho_{i j}^{2} \geq 1$. Hence (14) holds with $\tau_{0}=1$.

The follow theorem gives the rate of convergence over the parameter space $\mathcal{U}_{q}^{\star}$ under the spectral norm for the thresholding estimator $\hat{\Sigma}^{\star}(\delta)$.

Theorem 1 Let $\delta \geq 2$ and $0 \leq q<1$.

(i). Under (C1), we have, for some constant $C_{K_{1}, \delta, c, q}$ depending only on $\delta, c, q$ and $K_{1}$,

$$
\inf _{\Sigma_{0} \in \mathcal{U}_{q}^{\star}} P\left(\left\|\hat{\Sigma}^{\star}(\delta)-\Sigma_{0}\right\|_{2} \leq C_{K_{1}, \delta, c, q} s_{0}(p)\left(\frac{\log p}{n}\right)^{\frac{1-q}{2}}\right) \geq 1-O\left((\log p)^{-\frac{1}{2}} p^{-\delta+2}\right) .
$$


(ii). Under (C2), (16) holds with probability greater than $1-O\left((\log p)^{-1 / 2} p^{-\delta+2}+n^{-\epsilon / 8}\right)$.

Although $\mathcal{U}_{q}^{\star}$ is larger than the uniformity class $\mathcal{U}_{q}$, the rates of convergence of $\hat{\Sigma}^{\star}(\delta)$ over the two classes are of the same order $s_{0}(p)(\log p / n)^{(1-q) / 2}$.

Theorem 1 states the rate of convergence in terms of probability. The same rate of convergence holds in expectation with some additional mild assumptions. By (16) and some long but elementary calculations (see also the proof of Lemma 4), we have the following result on the mean squared spectral norm.

Proposition 1 Under (C1) and $p \geq n^{\xi}$ for some $\xi>0$, we have for $\delta \geq 7+\xi^{-1}, 0 \leq q<1$, and some constant $C>0$,

$$
\sup _{\boldsymbol{\Sigma}_{0} \in \mathcal{U}_{q}^{\star}} E\left\|\hat{\boldsymbol{\Sigma}}^{\star}(\delta)-\boldsymbol{\Sigma}_{0}\right\|_{2}^{2} \leq C s_{0}^{2}(p)\left(\frac{\log p}{n}\right)^{1-q}
$$

Remark 2 Cai and Zhou (2010) established the minimax rates of convergence under the spectral norm for sparse covariance matrix estimation over $\mathcal{U}_{q}$. It was shown that the optimal rate over $\mathcal{U}_{q}$ is $s_{0}(p)(\log p / n)^{(1-q) / 2}$. Since $\mathcal{U}_{q}\left(s_{0}(p)\right) \subseteq \mathcal{U}_{q}^{\star}\left(K^{1-q} s_{0}(p)\right)$, this implies immediately that the convergence rate attained by the adaptive thresholding estimator over $\mathcal{U}_{q}^{\star}$ in Theorem 1 and (17) is optimal.

Remark 3 The estimator $\hat{\mathbf{\Sigma}}^{\star}(\delta)$ yields immediately an estimate of the correlation matrix $\boldsymbol{R}_{0}=\left(r_{i j}\right)_{1 \leq i, j, \leq p}$ which is the object of direct interest in some statistical applications. Denote the corresponding estimator of $\boldsymbol{R}_{0}$ by $\hat{\boldsymbol{R}}^{\star}(\delta)=\left(\hat{r}_{i j}^{\star}\right)_{1 \leq i, j, \leq p}$ with $\hat{r}_{i j}^{\star}=\hat{\sigma}_{i j}^{\star} / \sqrt{\hat{\sigma}_{i i} \hat{\sigma}_{j j}}$. A parameter space for the correlation matrices is the following $\ell_{q}$ ball:

$$
\mathcal{R}_{q}^{\star}:=\mathcal{R}_{q}^{\star}\left(s_{0}(p)\right)=\left\{\boldsymbol{R}: \boldsymbol{R} \succ 0, \max _{i} \sum_{j=1}^{p}\left|r_{i j}\right|^{q} \leq s_{0}(p)\right\} .
$$

Then Theorem 1 holds for estimating the correlation matrix $\boldsymbol{R}_{0}$ by replacing $\hat{\boldsymbol{\Sigma}}^{\star}(\delta), \boldsymbol{\Sigma}_{0}$ and $\mathcal{U}_{q}^{\star}$ with $\hat{\boldsymbol{R}}^{\star}(\delta), \boldsymbol{R}_{0}$ and $\mathcal{R}_{q}^{\star}$, respectively. 
Note that the covariance matrix $\boldsymbol{\Sigma}_{0}$ can be written as $\boldsymbol{\Sigma}_{0}=\boldsymbol{D}^{1 / 2} \boldsymbol{R}_{0} \boldsymbol{D}^{1 / 2}$, where $\boldsymbol{D}=$ $\operatorname{diag}\left(\boldsymbol{\Sigma}_{0}\right)$. The covariance matrix can thus be viewed as a weighted version of the correlation matrix with weights $\left\{\left(\sigma_{i i} \sigma_{j j}\right)^{1 / 2}\right\}$. Correspondingly, the parameter space $\mathcal{U}_{q}^{\star}$ in (77) can be viewed as the weighted version of $\mathcal{R}_{q}^{\star}$ given in (18) with the same weights,

$$
\mathcal{U}_{q}^{\star}:=\left\{\boldsymbol{\Sigma}: \boldsymbol{\Sigma} \succ 0, \max _{i} \sum_{j=1}^{p}\left(\sigma_{i i} \sigma_{j j}\right)^{1 / 2}\left|r_{i j}\right|^{q} \leq s_{0}(p)\right\} .
$$

\subsection{Support recovery}

A closely related problem to estimating a sparse covariance matrix under spectral norm is the recovery of the support of the covariance matrix. This problem has been considered, for example, in Rothman, Levina and Zhu (2009). For support recovery, it is natural to consider the parameter space

$$
\overline{\mathcal{U}}_{0}:=\overline{\mathcal{U}}_{0}\left(s_{0}(p)\right)=\left\{\Sigma: \max _{i} \sum_{j=1}^{p} I\left\{\sigma_{i j} \neq 0\right\} \leq s_{0}(p)\right\},
$$

which assumes that the covariance matrix has at most $s_{0}(p)$ nonzero entries on each row.

Define the support of $\boldsymbol{\Sigma}_{0}=\left(\sigma_{i j}^{0}\right)$ by $\Psi=\left\{(i, j): \sigma_{i j}^{0} \neq 0\right\}$. The following theorem shows that the adaptive thresholding estimator $\hat{\Sigma}^{\star}(\delta)$ recovers the support $\Psi$ exactly with high probability when the magnitudes of nonzero entries are above certain threshold.

Theorem 2 Suppose that $\Sigma_{0} \in \overline{\mathcal{U}}_{0}$. Let $\delta \geq 2$ and

$$
\left|\sigma_{i j}^{0}\right|>(2+\delta+\gamma) \sqrt{\frac{\theta_{i j} \log p}{n}} \text { for all }(i, j) \in \Psi \text { and some } \gamma>0 \text {. }
$$

If either (C1) or (C2) holds, then we have

$$
\inf _{\boldsymbol{\Sigma}_{0} \in \overline{\mathcal{U}}_{0}} P\left(\operatorname{supp}\left(\hat{\boldsymbol{\Sigma}}^{\star}(\delta)\right)=\operatorname{supp}\left(\boldsymbol{\Sigma}_{0}\right)\right) \rightarrow 1
$$

Similar support recovery result was established for the generalized universal thresholding estimator in Rothman, Levina and Zhu (2009) under the condition $\max _{i} \sigma_{i i}^{0} \leq K$ and a lower bound condition similar to (19). Note that in Theorem 2, we do not require $\max _{i} \sigma_{i i} \leq K$. 
Following Rothman, Levina and Zhu (2009), the ability to recover the support can be evaluated via the true positive rate (TPR) in combination with the false positive rate (FPR), defined respectively as

$$
T P R=\frac{\#\left\{(i, j): \hat{\sigma}_{i j}^{\star} \neq 0 \text { and } \sigma_{i j} \neq 0\right\}}{\#\left\{(i, j): \sigma_{i j} \neq 0\right\}} \text { and } F P R=\frac{\#\left\{(i, j): \hat{\sigma}_{i j}^{\star} \neq 0 \text { and } \sigma_{i j}=0\right\}}{\#\left\{(i, j): \sigma_{i j}=0\right\}} .
$$

It follows from Theorem 2 directly that $\mathrm{P}(F P R=0) \rightarrow 1$ and $\mathrm{P}(T P R=1) \rightarrow 1$ under the conditions of the theorem.

The next result shows that $\delta=2$ is the optimal choice for support recovery in the sense that a thresholding estimator with any smaller choice of $\delta$ would fail to recover the support of $\boldsymbol{\Sigma}_{0}$ exactly with probability going to one. We assume $\mathbf{X}$ satisfies the following condition which is weaker than the Gaussian assumption.

(C3) Suppose that

$$
\mathrm{E}\left[\left(X_{i}-\mu_{i}\right)^{2}\left(X_{j}-\mu_{j}\right)\left(X_{k}-\mu_{k}\right)\right]=0, \quad \mathrm{E}\left[\left(X_{i}-\mu_{i}\right)\left(X_{j}-\mu_{j}\right)\left(X_{k}-\mu_{k}\right)\left(X_{l}-\mu_{l}\right)\right]=0
$$

if $\sigma_{j_{1} j_{2}}^{0}=0$ for all $j_{1} \neq j_{2} \in\{i, j, k, l\}$.

Theorem 3 Let $\lambda_{i j}=\tau \sqrt{\frac{\hat{\theta}_{i j} \log p}{n}}$ with $0<\tau<2$. Suppose that (C1) or (CQ) holds. Under (C3) and $p=\exp \left(o\left(n^{1 / 5}\right)\right)$, if $s_{0}(p)=O\left(p^{1-\tau_{1}}\right)$ with some $\tau^{2} / 4<\tau_{1}<1$ and $p \rightarrow \infty$, then

$$
\inf _{\Sigma_{0} \in \overline{\mathcal{U}}_{0}} P\left(\operatorname{supp}\left(\hat{\boldsymbol{\Sigma}}^{\star}(\tau)\right) \neq \operatorname{supp}\left(\boldsymbol{\Sigma}_{0}\right)\right) \rightarrow 1
$$

Remark 4 The condition $p=\exp \left(o\left(n^{1 / 5}\right)\right)$ is used in the proof to make sure the covariances of the samples $\left\{\mathbf{X}_{n}\right\}$ can be well approximated by normal vectors. It can be replaced by $p=\exp \left(o\left(n^{1 / 3}\right)\right)$ if $\mathbf{X}$ is a multivariate normal population.

\subsection{Comparison with universal thresholding}

It is interesting to compare the asymptotic results for adaptive thresholding estimator $\hat{\mathbf{\Sigma}}^{\star}(\delta)$ with the known results for universal thresholding estimators. We begin by comparing the 
rate of convergence of $\hat{\boldsymbol{\Sigma}}^{\star}(\delta)$ with that of the universal thresholding estimator $\hat{\boldsymbol{\Sigma}}_{u}$ introduced in Bickel and Levina (2008) in the case of polynomial-type tails. Suppose that (C2) holds. Bickel and Levina (2008) showed that

$$
\left\|\hat{\boldsymbol{\Sigma}}_{u}-\boldsymbol{\Sigma}_{0}\right\|_{2}=O_{\mathrm{P}}\left(s_{0}(p)\left(\frac{p^{1 /(1+\gamma+\epsilon / 2)}}{n^{1 / 2}}\right)^{1-q}\right)
$$

for $\boldsymbol{\Sigma}_{0} \in \mathcal{U}_{q}$. Clearly, the convergence rate given in Theorem 1 for the adaptive thresholding estimator is significantly faster than that in (20).

We next compare the rates over the class $\mathcal{U}_{q}^{\star}, 0 \leq q<1$. For brevity, we shall focus on the Gaussian case $\mathbf{X} \sim N\left(\boldsymbol{\mu}, \boldsymbol{\Sigma}_{0}\right)$. The following theorem gives the lower bound of the universal thresholding estimator.

Theorem 4 Assume that $n^{5 q} \leq p \leq \exp \left(o\left(n^{1 / 3}\right)\right)$ and $8 \leq s_{0}(p)<\min \left\{p^{1 / 4}, 4(n / \log p)^{1 / 2}\right\}$. We have, as $p \rightarrow \infty$,

$$
\inf _{\lambda_{n}} \sup _{\boldsymbol{\Sigma}_{0} \in \mathcal{U}_{q}^{\star}} P\left(\left\|\hat{\boldsymbol{\Sigma}}_{g}-\boldsymbol{\Sigma}_{0}\right\|_{2}>\frac{3}{64} s_{0}^{2-q}(p)\left(\frac{\log p}{n}\right)^{(1-q) / 2}\right) \rightarrow 1
$$

and hence for large $n$,

$$
\inf _{\lambda_{n}} \sup _{\boldsymbol{\Sigma}_{0} \in \mathcal{U}_{q}^{\star}} E\left\|\hat{\boldsymbol{\Sigma}}_{g}-\boldsymbol{\Sigma}_{0}\right\|_{2}^{2} \geq \frac{1}{512} s_{0}^{4-2 q}(p)\left(\frac{\log p}{n}\right)^{1-q} .
$$

The rate in (21) is slower than the optimal rate $s_{0}(p)(\log p / n)^{(1-q) / 2}$ given in (16) when $s_{0}(p) \rightarrow \infty$ as $p \rightarrow \infty$. Therefore no universal thresholding estimators can be minimax-rate optimal under the spectral norm over $\mathcal{U}_{q}^{\star}$ if $s_{0}(p) \rightarrow \infty$.

If we assume the mean of $\mathbf{X}$ is zero and ignore the term $\overline{\mathbf{X}}$ in $\boldsymbol{\Sigma}_{n}$, then the universal thresholding estimators given in Bickel and Levina (2008) and Rothman, Levina and Zhu (2009) use the sample mean of the samples $\left\{X_{k i} X_{k j} ; 1 \leq k \leq n\right\}$ to identify zero entries in the covariance matrix. The support of these estimators depends on the quantities $I\left\{\left|\hat{\sigma}_{i j}\right| \geq\right.$ $\left.\lambda_{n}\right\}$. In the high dimensional setting, the sample mean is usually unstable for non-Gaussian distributions with heavier tails. Non-Gaussian data can often arise from many practical 
applications such as in finance and genomics. For our estimator, instead of the sample mean, we use the Student $t$ statistic $\hat{\sigma}_{i j} / \hat{\theta}_{i j}^{1 / 2}$ to distinguish zero and nonzero entries. Our

support recovery depends on the quantities $I\left\{\left|\hat{\sigma}_{i j}\right| / \hat{\theta}_{i j}^{1 / 2} \geq 2 \sqrt{\log p / n}\right\}$ which are more stable than $I\left\{\left|\hat{\sigma}_{i j}\right| \geq \lambda_{n}\right\}$, since $t$ statistic is much more stable than the sample mean; see Shao (1999) for the theoretical justification.

\section{Data-driven choice of $\delta$}

Section 3 analyzes the properties of the adaptive thresholding estimator with a fixed value of $\delta$. Alternatively, $\delta$ can be selected empirically through cross validation (CV). In Bickel and Levina (2008) the value of the universal thresholding level $\lambda_{n}$ is not fully specified and the CV method was used to select $\lambda_{n}$ empirically. They obtained the convergence rate under the Frobenius norm for an estimator that is based only on partial samples. Theoretical analysis on the rate of convergence under the spectral norm is still lacking. In this section, we first briefly describe the $\mathrm{CV}$ method for choosing $\delta$ and then derive the theoretical properties of the resulting estimator under the spectral norm.

Divide the sample $\left\{\mathbf{X}_{k} ; 1 \leq k \leq n\right\}$ into two subsamples at random. Let $n_{1}$ and $n_{2}=n-n_{1}$ be the two sample sizes for the random split satisfying $n_{1} \asymp n_{2} \asymp n$, and let $\hat{\Sigma}_{1}^{v}, \hat{\Sigma}_{2}^{v}$ be the two sample covariance matrices from the $v$ th split, for $v=1, \ldots, H$, where $H$ is a fixed integer. Let $\hat{\boldsymbol{\Sigma}}_{1}^{\star v}(\delta)$ and $\hat{\boldsymbol{\Sigma}}_{2}^{\star v}(\delta)$ be defined as in (11) from the $v$ th split and

$$
\hat{R}(\delta)=\frac{1}{H} \sum_{v=1}^{H}\left\|\hat{\boldsymbol{\Sigma}}_{1}^{\star v}(\delta)-\hat{\mathbf{\Sigma}}_{2}^{v}\right\|_{F}^{2} .
$$

Let $a_{j}=j / N, 0 \leq j \leq 4 N$ be $4 N+1$ points in $[0,4]$ and take

$$
\hat{\delta}=\hat{j} / N, \quad \text { where } \quad \hat{j}=\underset{0 \leq j \leq 4 N}{\arg \min } \hat{R}(j / N),
$$

where $N>0$ is a fixed integer. If there are several $j$ attain the minimum value, $\hat{j}$ is chosen to be the smallest one. The final estimator of the covariance matrix $\boldsymbol{\Sigma}_{0}$ is given by $\hat{\Sigma}^{\star}(\hat{\delta})$. 
Theorem 5 Suppose $\boldsymbol{X} \sim N\left(\boldsymbol{\mu}, \boldsymbol{\Sigma}_{0}\right)$ with $\boldsymbol{\Sigma}_{0} \in \mathcal{U}_{0}$ and $\min _{i} \sigma_{i i}^{0} \geq \tau_{0}$ for some $\tau_{0}>0$. Let $s_{0}(p)=O\left((\log p)^{\gamma}\right)$ for some $\gamma<1$ and $n^{\xi} \leq p \leq \exp \left(o\left(n^{1 / 3}\right)\right)$ for some $\xi>0$. We have

$$
\inf _{\boldsymbol{\Sigma}_{0} \in \mathcal{U}_{0}} P\left(\left\|\hat{\boldsymbol{\Sigma}}^{\star}(\hat{\delta})-\boldsymbol{\Sigma}_{0}\right\|_{2} \leq C s_{0}(p)\left(\frac{\log p}{n}\right)^{1 / 2}\right) \rightarrow 1
$$

Remark 5 The assumption that $N$ is fixed is not a stringent condition since we only consider $\delta$ belonging to the fixed interval $[0,4]$. Moreover, we will only focus on the matrices in $\mathcal{U}_{0}$ due to the complexity of the proof. Extending to the case $N \rightarrow \infty$ with certain rate and more general $\boldsymbol{\Sigma}_{0}$ is possible. However, it requires far more complicated proof and will not be discussed in the present paper.

Remark 6 The condition $s_{0}(p)=O\left((\log p)^{\gamma}\right)$ used in the theorem is purely for technical reasons and we believe that it is not essentially needed and can be weakened. This condition is not stringent when $p=\exp \left(n^{\alpha}\right)$ and it becomes restrictive if $p=O\left(n^{\alpha}\right)$.

Similar to the fixed $\delta$ case, we also consider support recovery with the estimator $\hat{\boldsymbol{\Sigma}}^{\star}(\hat{\delta})$.

Proposition 2 Suppose the conditions in Theorem 5 hold. For $\hat{\mathbf{\Sigma}}^{\star}(\hat{\delta})$, we have

$$
F P R=O_{P}\left(s_{0}(p) / p\right) \rightarrow 0
$$

Moreover, since $\hat{\delta} \leq 4$, we have TPR $=1$ with probability tending to one if the lower bound in (19) holds with $2+\delta$ being replaced by 6 .

\section{$5 \quad$ Numerical Results}

The adaptive thresholding procedure presented in Section 2 is easy to implement. In this section, the numerical performance of the proposed adaptive thresholding estimator $\hat{\Sigma}^{\star}(\delta)$ is studied using Monte Carlo simulations. Both methods for choosing the regularization 
parameter $\delta$ are considered and their performance are compared with that of universal thresholding estimators. The adaptive thresholding estimator is illustrated in an analysis on a dataset from a small round blue-cell tumors microarray experiment.

\subsection{Simulation}

The following two types of sparse covariance matrices are considered in the simulations to investigate the numerical properties of the adaptive thresholding estimator $\hat{\mathbf{\Sigma}}^{\star}(\delta)$.

- Model 1 (banded matrix with ordering). $\boldsymbol{\Sigma}_{0}=\operatorname{diag}\left(\boldsymbol{A}_{1}, \boldsymbol{A}_{2}\right)$, where $\boldsymbol{A}_{1}=\left(\sigma_{i j}\right)_{1 \leq i, j \leq p / 2}$, $\sigma_{i j}=\left(1-\frac{|i-j|}{10}\right)_{+}, \boldsymbol{A}_{2}=4 \boldsymbol{I}_{p / 2 \times p / 2} . \boldsymbol{\Sigma}_{0}$ is a two-block diagonal matrix. $\boldsymbol{A}_{1}$ is a banded and sparse covariance matrix. $\boldsymbol{A}_{2}$ is a diagonal matrix with 4 along the diagonal.

- Model 2 (sparse matrix without ordering). $\boldsymbol{\Sigma}_{0}=\operatorname{diag}\left(\boldsymbol{A}_{1}, \boldsymbol{A}_{2}\right)$, where $\boldsymbol{A}_{2}=4 \boldsymbol{I}_{p / 2 \times p / 2}$, $\boldsymbol{A}_{1}=\boldsymbol{B}+\epsilon \boldsymbol{I}_{p / 2 \times p / 2}, \boldsymbol{B}=\left(b_{i j}\right)_{p / 2 \times p / 2}$ with independent $b_{i j}=\operatorname{unif}(0.3,0.8) \times \operatorname{Ber}(1,0.2)$. Here unif $(0.3,0.8)$ is a random variable taking value uniformly in $[0.3,0.8]$; $\operatorname{Ber}(1,0.2)$ is a Bernoulli random variable which takes value 1 with probability 0.2 and 0 with probability 0.8; and $\epsilon=\max \left(-\lambda_{\min }(\boldsymbol{B}), 0\right)+0.01$ to ensure that $\boldsymbol{A}_{1}$ is positive definite.

Under each model, $n=100$ independent and identically distributed $p$-variate random vectors are generated from the normal distribution with mean 0 and covariance matrix $\boldsymbol{\Sigma}_{0}$, for $p=30,100,200$. In each setting, 100 replications are used. We compare the numerical performance between the adaptive thresholding estimators $\hat{\boldsymbol{\Sigma}}^{\star}(\hat{\delta})$ and $\hat{\mathbf{\Sigma}}_{2}^{\star} \equiv \hat{\mathbf{\Sigma}}^{\star}(2)$ and with the universal thresholding estimator $\hat{\boldsymbol{\Sigma}}_{g}$ of Rothman, Levina and Zhu (2009). Here

$\hat{\delta}$ is selected by five fold cross-validation in Section $4, \hat{\Sigma}_{2}^{\star}$ is the adaptive thresholding estimator with fixed $\delta=2$. The thresholding level $\lambda_{n}$ in $\hat{\Sigma}_{g}$ is selected by five fold crossvalidation method used in Bickel and Levina (2008). For each procedure, we consider two types of thresholding functions, the hard thresholding and the adaptive lasso thresholding 
$s_{\lambda}(z)=x\left(1-|\lambda / x|^{\eta}\right)$ with $\eta=4$. The losses are measured by three matrix norms: the spectral norm, the matrix $\ell_{1}$ norm and the Frobenius norm. We report in Tables 11 and 2 the means and standard errors of these losses. We also carried out simulations with the SCAD thresholding function for both universal thresholding and adaptive thresholding. The phenomenon is very similar. The SCAD adaptive thresholding also outperforms the SCAD universal thresholding. For reasons of space, the results are not reported here.

Table 1: Comparison of average matrix losses for Model 1 over 100 replications. The standard errors are given in the parentheses.

\begin{tabular}{|c|c|c|c|c|c|c|}
\hline \multicolumn{4}{|c|}{ Adaptive lasso } & \multicolumn{3}{|c|}{ Hard } \\
\hline$p$ & $\hat{\Sigma}_{g}$ & $\hat{\boldsymbol{\Sigma}}^{\star}(\hat{\delta})$ & $\hat{\Sigma}_{2}^{\star}$ & $\hat{\Sigma}_{g}$ & $\hat{\boldsymbol{\Sigma}}^{\star}(\hat{\delta})$ & $\hat{\boldsymbol{\Sigma}}_{2}^{\star}$ \\
\hline \multicolumn{7}{|c|}{ Operator norm } \\
\hline 30 & $3.53(0.13)$ & $1.72(0.05)$ & $2.39(0.07)$ & $3.50(0.14)$ & $1.77(0.05)$ & $1.77(0.04)$ \\
\hline 100 & $7.94(0.11)$ & $2.72(0.05)$ & $4.68(0.06)$ & $8.64(0.07)$ & $2.57(0.05)$ & $3.04(0.05)$ \\
\hline 200 & $8.95(0.004)$ & $3.23(0.05)$ & $5.70(0.05)$ & $8.95(0.004)$ & $3.02(0.05)$ & $3.77(0.05)$ \\
\hline \multicolumn{7}{|c|}{ Matrix $\ell_{1}$ norm } \\
\hline 30 & $5.29(0.15)$ & $2.57(0.08)$ & $3.34(0.09)$ & $5.71(0.15)$ & $2.60(0.09)$ & $2.70(0.06)$ \\
\hline 100 & $9.03(0.05)$ & $4.15(0.07)$ & $6.39(0.09)$ & $9.24(0.03)$ & $4.17(0.07)$ & $4.87(0.09)$ \\
\hline 200 & $9.35(0.01)$ & $4.90(0.07)$ & $7.64(0.07)$ & $9.35(0.01)$ & $4.89(0.07)$ & $5.97(0.09)$ \\
\hline \multicolumn{7}{|c|}{ Frobenius norm } \\
\hline 30 & $5.97(0.10)$ & $3.15(0.05)$ & $3.68(0.05)$ & $6.58(0.09)$ & $3.29(0.05)$ & $3.29(0.04)$ \\
\hline 100 & $15.93(0.12)$ & $6.57(0.05)$ & $8.92(0.06)$ & $16.88(0.03)$ & $6.79(0.06)$ & $7.53(0.05)$ \\
\hline 200 & $24.23(0.01)$ & $9.62(0.05)$ & $14.20(0.07)$ & $24.24(0.01)$ & $9.97(0.06)$ & $11.68(0.05)$ \\
\hline
\end{tabular}


Table 2: Comparison of average matrix losses for Model 2 over 100 replications. The standard errors are given in the parentheses.

\begin{tabular}{|ccccccc|}
\hline \multicolumn{7}{|c|}{ Adaptive lasso } \\
\hline$p$ & $\hat{\mathbf{\Sigma}}_{g}$ & $\hat{\mathbf{\Sigma}}^{\star}(\hat{\delta})$ & $\hat{\mathbf{\Sigma}}_{2}^{\star}$ & $\hat{\mathbf{\Sigma}}_{g}$ & $\hat{\mathbf{\Sigma}}^{\star}(\hat{\delta})$ & $\hat{\mathbf{\Sigma}}_{2}^{\star}$ \\
\hline \multicolumn{7}{|c}{ Operator norm } \\
30 & $1.48(0.02)$ & $1.24(0.03)$ & $1.19(0.03)$ & $1.50(0.02)$ & $1.25(0.03)$ & $1.21(0.03)$ \\
100 & $5.31(0.01)$ & $2.82(0.05)$ & $4.71(0.03)$ & $5.31(0.01)$ & $2.69(0.05)$ & $3.97(0.04)$ \\
200 & $10.74(0.01)$ & $6.78(0.08)$ & $10.52(0.02)$ & $10.74(0.01)$ & $6.58(0.10)$ & $10.04(0.03)$ \\
& & & Matrix $\ell_{1}$ & norm & & \\
30 & $1.70(0.03)$ & $1.33(0.04)$ & $1.22(0.03)$ & $1.70(0.02)$ & $1.32(0.04)$ & $1.24(0.03)$ \\
100 & $6.16(0.01)$ & $4.10(0.05)$ & $5.52(0.03)$ & $6.16(0.01)$ & $4.20(0.06)$ & $5.22(0.03)$ \\
200 & $12.70(0.01)$ & $9.81(0.08)$ & $12.31(0.04)$ & $12.70(0.01)$ & $10.06(0.08)$ & $12.06(0.04)$ \\
& & & & & & \\
30 & $4.08(0.03)$ & $2.52(0.04)$ & $2.57(0.04)$ & $4.10(0.03)$ & $2.50(0.04)$ & $2.45(0.04)$ \\
100 & $12.77(0.01)$ & $7.57(0.05)$ & $10.96(0.04)$ & $12.78(0.02)$ & $8.07(0.06)$ & $10.00(0.05)$ \\
200 & $25.51(0.01)$ & $16.94(0.07)$ & $24.67(0.03)$ & $25.52(0.01)$ & $18.69(0.07)$ & $24.05(0.03)$ \\
\hline
\end{tabular}


Under Model 1 and Model 2, both adaptive thresholding estimators $\hat{\mathbf{\Sigma}}^{\star}(\hat{\delta})$ and $\hat{\mathbf{\Sigma}}_{2}^{\star}$ uniformly outperform the universal thresholding rule $\hat{\Sigma}_{g}$ significantly, regardless which thresholding function or which loss function is used. Between $\hat{\Sigma}^{\star}(\hat{\delta})$ and $\hat{\Sigma}_{2}^{\star}, \hat{\Sigma}^{\star}(\hat{\delta})$ performs better than $\hat{\Sigma}_{2}^{\star}$ in general. Between the two thresholding functions, the hard thresholding rule outperforms the adaptive lasso thresholding rule for $\hat{\mathbf{\Sigma}}_{2}^{\star}$, while the difference is not significant for $\hat{\Sigma}^{\star}(\hat{\delta})$. For both models, the behaviors of hard and adaptive lasso universal thresholding rules are very similar. They both tend to "over-threshold" and remove many nonzero off-diagonal entries of the covariance matrices.

For support recovery, again both $\hat{\boldsymbol{\Sigma}}^{\star}(\hat{\delta})$ and $\hat{\boldsymbol{\Sigma}}_{2}^{\star}$ outperform $\hat{\boldsymbol{\Sigma}}_{g}$. The values of TPR and FPR based on the off-diagonal entries are reported in Tables 3 and 4 . For Model 1, $\hat{\Sigma}_{g}$ tends to estimate many nonzero off-diagonal entries by zero when $p$ is large. To better illustrate the recovery performance elementwise for the two models, the heat maps of the nonzeros identified out of 100 replications when $p=60$ are pictured in Figures 1 and 2. The heat maps suggest that the sparsity patterns recovered by $\hat{\Sigma}^{\star}(\hat{\delta})$ and $\hat{\Sigma}_{2}^{\star}$ have significantly better resemblance to the true model than $\hat{\Sigma}_{g}$.

Table 3: Comparison of support recovery for Model 1 over 100 replications.

\begin{tabular}{|rrrrrrrrr|}
\hline \multicolumn{4}{|c}{ Adaptive lasso } & \multicolumn{3}{c|}{ Hard } \\
\hline$p$ & & $\hat{\boldsymbol{\Sigma}}_{g}$ & $\hat{\boldsymbol{\Sigma}}^{\star}(\hat{\delta})$ & $\hat{\boldsymbol{\Sigma}}_{2}^{\star}$ & $\hat{\boldsymbol{\Sigma}}_{g}$ & $\hat{\mathbf{\Sigma}}^{\star}(\hat{\delta})$ & $\hat{\mathbf{\Sigma}}_{2}^{\star}$ \\
30 & TPR & 0.57 & 0.84 & 0.72 & 0.46 & 0.79 & 0.72 \\
& FPR & 0.07 & 0.01 & 0.00 & 0.05 & 0.003 & 0.00 \\
100 & TPR & 0.15 & 0.76 & 0.57 & 0.008 & 0.69 & 0.57 \\
& FPR & 0.01 & 0.01 & 0.00 & 0.00 & 0.00 & 0.00 \\
200 & TPR & 0.00 & 0.73 & 0.51 & 0.00 & 0.65 & 0.51 \\
FPR & 0.00 & 0.003 & 0.00 & 0.00 & 0.00 & 0.00 \\
\hline
\end{tabular}


Table 4: Comparison of support recovery for Model 2 over 100 replications.

\begin{tabular}{|c|c|c|c|c|c|c|}
\hline \multicolumn{4}{|c|}{ Adaptive lasso } & \multicolumn{3}{|c|}{ Hard } \\
\hline$p$ & $\hat{\boldsymbol{\Sigma}}_{g}$ & $\hat{\boldsymbol{\Sigma}}^{\star}(\hat{\delta})$ & $\hat{\boldsymbol{\Sigma}}_{2}^{\star}$ & $\hat{\boldsymbol{\Sigma}}_{g}$ & $\hat{\boldsymbol{\Sigma}}^{\star}(\hat{\delta})$ & $\hat{\boldsymbol{\Sigma}}_{2}^{\star}$ \\
\hline 30 & TPR 0.02 & 0.95 & 0.88 & 0.00 & 0.91 & 0.88 \\
\hline & FPR 0.00 & 0.01 & 0.00 & 0.00 & 0.00 & 0.00 \\
\hline 100 & TPR 0.00 & 0.80 & 0.33 & 0.00 & 0.66 & 0.33 \\
\hline & FPR 0.00 & 0.01 & 0.00 & 0.00 & 0.00 & 0.00 \\
\hline 200 & TPR 0.00 & 0.68 & 0.09 & 0.00 & 0.49 & 0.09 \\
\hline & FPR 0.00 & 0.01 & 0.00 & 0.00 & 0.00 & 0.00 \\
\hline
\end{tabular}

Model 1

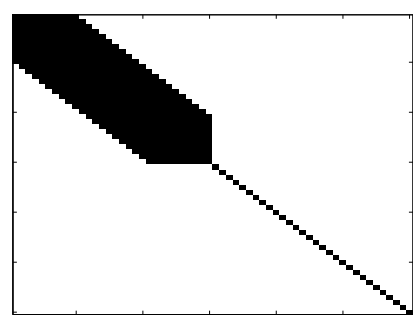

(a) Truth

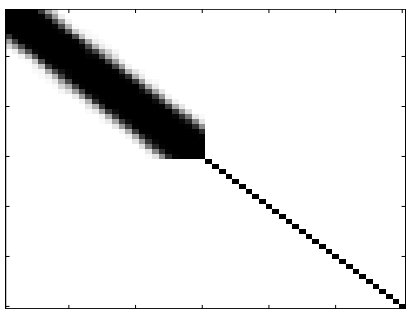

(d) $\hat{\boldsymbol{\Sigma}}_{2}^{\star}$

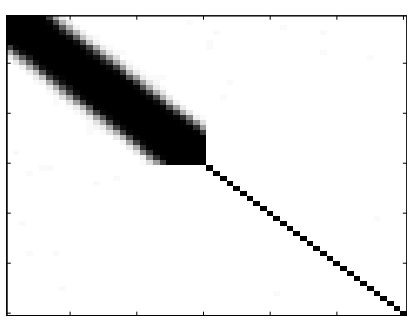

(b) $\hat{\boldsymbol{\Sigma}}(\hat{\delta})($ Hard $)$

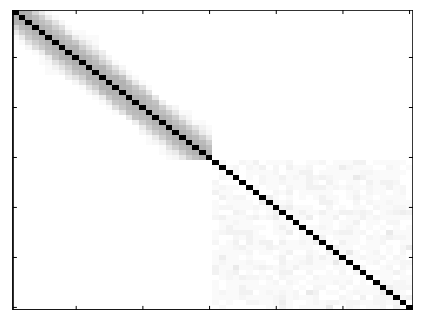

(e) $\hat{\boldsymbol{\Sigma}}_{g}($ Hard $)$

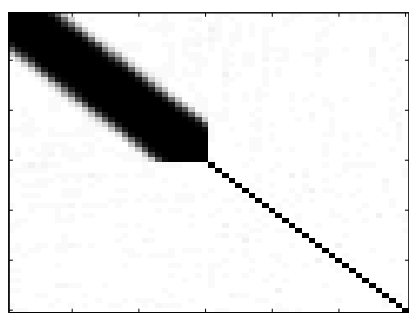

(c) $\hat{\boldsymbol{\Sigma}}(\hat{\delta})($ Adap.lasso $)$

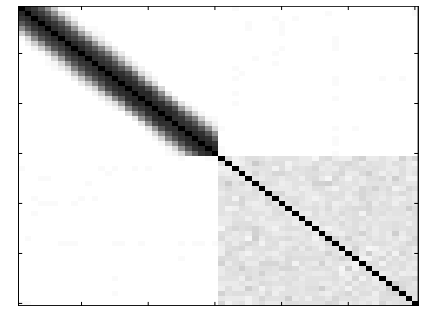

(f) $\hat{\boldsymbol{\Sigma}}_{g}$ (Adap.lasso)

Figure 1: Heat maps of the frequency of the zeros identified for each entry of the covariance matrix (when $p=60$ ) out of 100 replications. White color is 100 zeros identified out of 100 runs, and black is $0 / 100$. 
Model 2

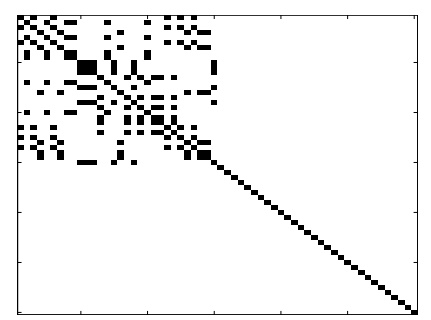

(a) Truth

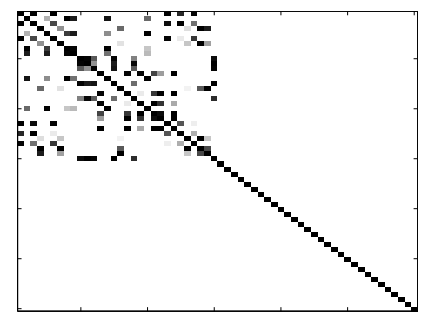

(d) $\hat{\Sigma}_{2}^{\star}$

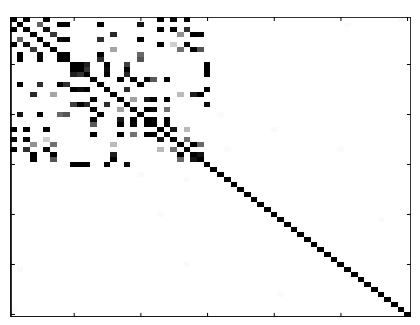

(b) $\hat{\boldsymbol{\Sigma}}(\hat{\delta})($ Hard $)$

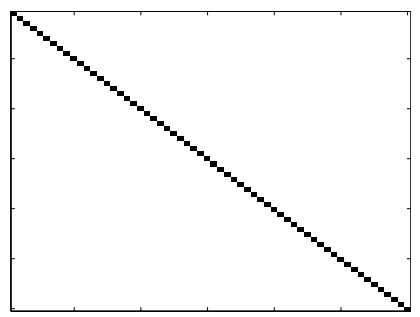

(e) $\hat{\boldsymbol{\Sigma}}_{g}($ Hard $)$

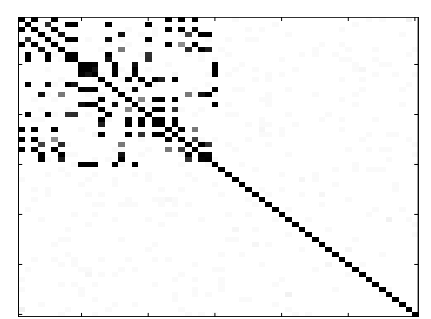

(c) $\hat{\boldsymbol{\Sigma}}(\hat{\delta})$ (Adap.lasso)

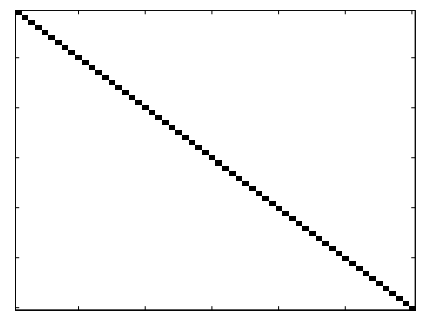

(f) $\hat{\boldsymbol{\Sigma}}_{g}$ (Adap.lasso)

Figure 2: Heat maps of the frequency of the zeros identified for each entry of the covariance matrix (when $p=60$ ) out of 100 replications. White color is 100 zeros identified out of 100 runs, and black is $0 / 100$. 


\subsection{Correlation analysis on real data}

We now apply the adaptive thresholding estimator $\hat{\mathbf{\Sigma}}^{\star}(\delta)$ to a dataset from a small round blue-cell tumors (SRBC) microarray experiment (Khan et al., 2001) and compare the ability of support recovery with that of the universal thresholding estimator $\hat{\boldsymbol{\Sigma}}_{g}$. The estimator $\hat{\Sigma}_{2}^{\star}$ is not considered here since the simulation results in Section 5.1] show that $\hat{\Sigma}^{\star}(\hat{\delta})$ outperforms $\hat{\Sigma}_{2}^{\star}$ when the sample size is not large. The SRBC data set has been analyzed in Rothman, Levina and Zhu (2009) in which the universal thresholding rules were considered. To make the results comparable, we shall follow the same steps as those in Rothman, Levina and Zhu (2009).

The SRBC data has 63 training tissue samples, and 2308 gene expression values recorded for each sample. The original data has 6567 genes and was reduced to 2308 genes after an initial filtering; see Khan et al. (2001). The 63 tissue samples contain four types of tumors (23 EWS, 8 BL-NHL, 12 NB, and 20 RMS). As in Rothman, Levina and Zhu (2009), the genes were first ranked by the amount of discriminative information based on the F-statistic,

$$
F=\frac{\frac{1}{k-1} \sum_{m=1}^{k} n_{m}\left(\bar{x}_{m}-\bar{x}\right)^{2}}{\frac{1}{n-k} \sum_{m=1}^{k}\left(n_{m}-1\right) \hat{\sigma}_{m}^{2}},
$$

where $n=63$ is the sample size, $k=4$ is the number of classes, $n_{m}, 1 \leq m \leq 4$, are the sample sizes of the four types of tumors, $\bar{x}_{m}$ and $\hat{\sigma}_{m}$ are the sample mean and sample variance of the class $m$, and $\bar{x}$ is the overall sample mean. According to the $F$ values, the top 40 and bottom 160 genes were chosen. The first 40 genes were also ordered according to the ordering given in Rothman, Levina and Zhu (2009). Based on the 200 genes, the performance of the two estimators $\hat{\Sigma}^{\star}(\hat{\delta})$ and $\hat{\Sigma}_{g}$ was considered. The tuning parameters $\hat{\delta}$ and $\lambda_{n}$ were selected by five fold cross validation. To this end, we need to divide the 63 samples into five groups of nearly equal sizes. As there are four types of tumors in the samples, we let the proportions of the four types of tumors in each group be nearly equal 
so that each fold is a good representative of the whole. Three fold cross validation is also used in this way and the results are similar.

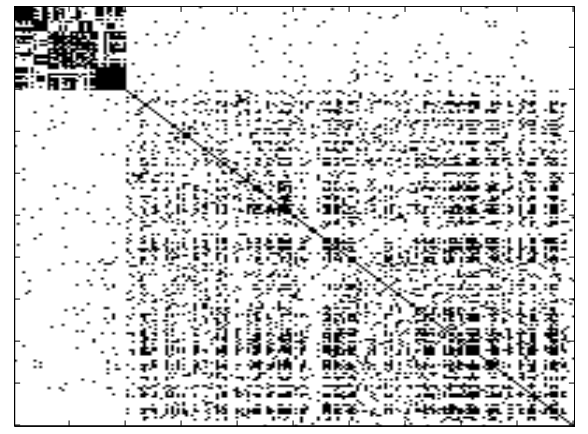

(a) $\hat{\boldsymbol{\Sigma}}(\hat{\delta})$ Hard $(83.11 \%$ zeros $)$

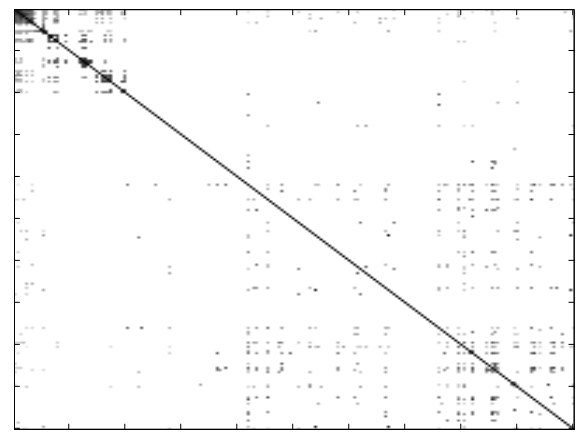

(c) $\hat{\boldsymbol{\Sigma}}_{g}$ Hard $(97.88 \%$ zeros $)$

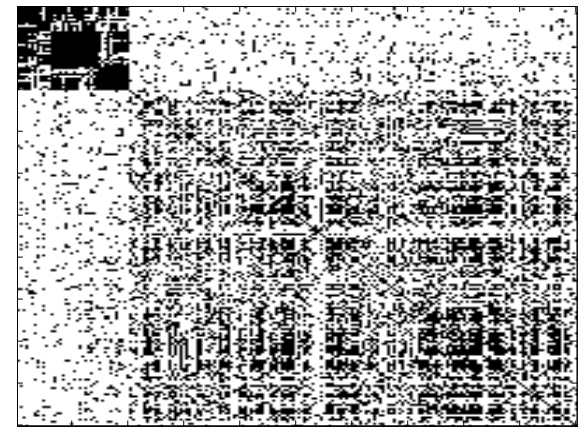

(b) $\hat{\boldsymbol{\Sigma}}(\hat{\delta})$ AL $(69.78 \%$ zeros $)$

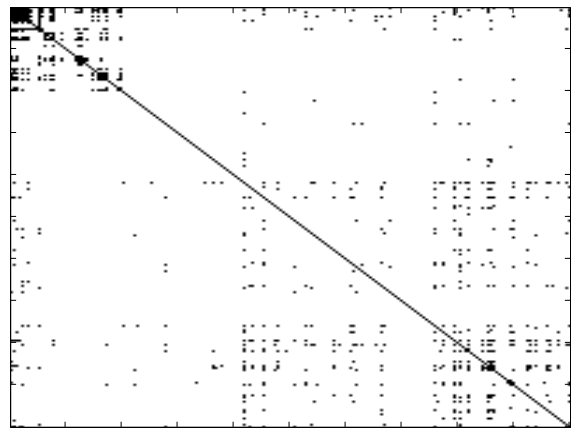

(d) $\hat{\boldsymbol{\Sigma}}_{g}$ AL $(97.88 \%$ zeros $)$

Figure 3: Heatmaps of the estimated supports.

Figure 3 plots the heat maps of $\hat{\Sigma}^{\star}(\hat{\delta})$ with hard thresholding $\left(\hat{\Sigma}^{\star}(\hat{\delta})\right.$ Hard $), \hat{\Sigma}_{g}$ with hard thresholding $\left(\hat{\boldsymbol{\Sigma}}_{g} \operatorname{Hard}\right), \hat{\boldsymbol{\Sigma}}^{\star}(\hat{\delta})$ with adaptive lasso thresholding $\left(\hat{\boldsymbol{\Sigma}}^{\star}(\hat{\delta})\right.$ AL), $\hat{\boldsymbol{\Sigma}}_{g}$ with adaptive lasso thresholding $\left(\hat{\Sigma}_{g} \mathrm{AL}\right) . \hat{\boldsymbol{\Sigma}}_{g}$ AL and $\hat{\boldsymbol{\Sigma}}_{g}$ Hard result in very sparse estimators, with $97.88 \%$ zero elements in off diagonal positions. The estimator $\hat{\Sigma}^{\star}(\hat{\delta})$ AL is the least sparse one with $69.78 \%$ zeros, while $\hat{\Sigma}^{\star}(\hat{\delta})$ Hard has $83.11 \%$ zeros. The "over-threshold" phenomenon in the real data analysis is consistent with that observed in the simulations. The universal thresholding rule removes many nonzero off diagonal entries and results in an "over-sparse" estimate, while adaptive thresholding with different individual levels results in a clean but more informative estimate of the sparsity structure. 


\section{Discussion}

This paper introduces an adaptive entry-dependent thresholding procedure for estimating sparse covariance matrices. The proposed estimator $\hat{\mathbf{\Sigma}}^{\star}(\delta)=\left(\hat{\sigma}_{i j}^{\star}\right)$ enjoys excellent performance both theoretically and numerically. In particular, $\hat{\Sigma}^{\star}(\delta)$ attains the optimal rate of convergence over $\mathcal{U}_{q}^{\star}$ given in (7) while universal thresholding estimators are shown to be sub-optimal. The main reason that universal thresholding does not perform well is that the sample covariances can have a wide range of variabilities. A simple and natural way to deal with the heteroscedasticity is to first estimate the correlation matrix $\boldsymbol{R}_{0}$ and then renormalize by the sample variances to obtain an estimate of the covariance matrix. We shall discuss below two approaches based on this idea.

Denote the sample correlation matrix by $\hat{\boldsymbol{R}}=\left(\hat{r}_{i j}\right)_{1 \leq i, j \leq p}$ with $\hat{r}_{i, j}=\hat{\sigma}_{i j} / \sqrt{\hat{\sigma}_{i i} \hat{\sigma}_{j j}}$. An estimate of the correlation matrix $\boldsymbol{R}_{0}$ can be obtained by thresholding $\hat{r}_{i j}$. Define the universal thresholding estimator of the correlation matrix by $\hat{\boldsymbol{R}}\left(\lambda_{n}\right)=\left(\hat{r}_{i j}^{t h r}\right)_{p \times p}$ with

$$
\hat{r}_{i j}^{t h r}=\hat{r}_{i j} I\left\{\left|\hat{r}_{i j}\right| \geq \lambda_{n}\right\}
$$

and the corresponding estimator of the covariance matrix by $\hat{\boldsymbol{\Sigma}}_{R}=\boldsymbol{D}_{n}^{1 / 2} \hat{\boldsymbol{R}}\left(\lambda_{n}\right) \boldsymbol{D}_{n}^{1 / 2}$, where $\boldsymbol{D}_{n}=\operatorname{diag}\left(\boldsymbol{\Sigma}_{n}\right)$. It is easy to see that a good choice of the threshold $\lambda_{n}$ is $\lambda_{n}=C \sqrt{(\log p) / n}$ for some constant $C>0$. It is however difficult to choose $C$ because the choice depends on the unknown underlying distribution. Assuming the constant $C$ is chosen sufficiently large, it can be shown that the resulting estimator $\hat{\boldsymbol{\Sigma}}_{R}$ attains the same minimax rate of convergence. However, the estimator $\hat{\boldsymbol{\Sigma}}_{R}$ is less efficient than $\hat{\boldsymbol{\Sigma}}^{\star}(\delta)$ for support recovery. In fact, $\hat{\Sigma}_{R}$ is unable to recover the support of $\boldsymbol{\Sigma}_{0}$ exactly for a class of non-Gaussian distributions of $\mathbf{X}$. Denote by $\mathcal{V}\left(\gamma, \delta, K_{1}\right)$ the class of distributions $F$ of $\mathbf{X}$ satisfying the conditions of Theorem 2. Then it can be shown that for any $\gamma>0, \delta \geq 2$ and some $K_{1}=K_{1}(\gamma)>0$

$$
\inf _{\lambda_{n}} \sup _{F \in \mathcal{V}\left(\gamma, \delta, K_{1}\right)} \mathrm{P}\left(\operatorname{supp}\left(\hat{\boldsymbol{\Sigma}}_{R}\right) \neq \operatorname{supp}\left(\boldsymbol{\Sigma}_{0}\right)\right) \rightarrow 1
$$


The sample correlation coefficients $\hat{r}_{i j}$ are not homoscedastic, although the range of variabilities is smaller in comparison to that of sample covariances. This is in fact the main reason for the negative result on support recovery given in Equation (23). A natural approach to deal with the heteroscedasticity of the sample correlation coefficients is to first stabilize the variance by using Fisher's z-transformation, then threshold and finally obtain the estimator by inverse transform. Applying Fisher's z-transformation to each correlation coefficient yields

$$
\hat{Z}_{i j}=\frac{1}{2} \ln \frac{1+\hat{r}_{i j}}{1-\hat{r}_{i j}} .
$$

When $\mathbf{X}$ is multivariate normal, it is well-known that $\hat{Z}_{i j}$ is asymptotically normal with mean $(1 / 2) \ln \left(\left(1+r_{i j}\right) /\left(1-r_{i j}\right)\right)$ and variance $1 /(n-3)$. The behavior of $\hat{Z}_{i j}$ in the nonGaussian case is more complicated. In general, the asymptotic variance of $\hat{Z}_{i j}$ depends on $\mathrm{E} X_{i}^{2} X_{j}^{2}$ even when $r_{i j}=0$; see Hawkins (1989). Similar to the method of thresholding the

sample correlation coefficients discussed earlier, universally thresholding $\left(\hat{Z}_{i j}\right)_{p \times p}$ is unable to recover the support of $\boldsymbol{\Sigma}_{0}$ exactly for a class of non-Gaussian distributions of $\mathbf{X}$ satisfying the conditions in Theorem 2 ,

In conclusion, the two natural approaches based on the sample correlation matrix discussed above are not as efficient as the entry-dependent thresholding method we proposed in Section 2, For reasons of space, we omit the proofs of the results stated in this section. We shall explore these issues in detail elsewhere.

\section{Proofs}

We begin by collecting a few technical lemmas which are essential for the proofs of the main results. The first lemma is an exponential inequality on the partial sums of independent random variables. 
Lemma 1 Let $\xi_{1}, \cdots, \xi_{n}$ be independent random variables with mean zero. Suppose that there exists some $t>0$ and $\bar{B}_{n}$ such that $\sum_{k=1}^{n} E \xi_{k}^{2} e^{t\left|\xi_{k}\right|} \leq \bar{B}_{n}^{2}$. Then for $0<x \leq \bar{B}_{n}$,

$$
P\left(\sum_{k=1}^{n} \xi_{k} \geq C_{t} \bar{B}_{n} x\right) \leq \exp \left(-x^{2}\right)
$$

where $C_{t}=t+t^{-1}$.

Proof of Lemma 1. By the inequality $\left|e^{s}-1-s\right| \leq s^{2} e^{s \max (s, 0)}$, we have for any $t \geq 0$,

$$
\begin{aligned}
\mathrm{P}\left(\sum_{k=1}^{n} \xi_{k} \geq C_{K} \bar{B}_{n} x\right) & \leq \exp \left(-t C_{\eta} \bar{B}_{n} x\right) \prod_{k=1}^{n} \mathrm{E} \exp \left(t \xi_{k}\right) \\
& \leq \exp \left(-t C_{\eta} \bar{B}_{n} x\right) \prod_{k=1}^{n}\left(1+t^{2} \mathrm{E} \xi_{k}^{2} e^{t\left|\xi_{k}\right|}\right) \\
& \leq \exp \left(-t C_{\eta} \bar{B}_{n} x+\sum_{k=1}^{n} t^{2} \mathrm{E} \xi_{k}^{2} e^{t\left|\xi_{k}\right|}\right)
\end{aligned}
$$

Take $t=\eta\left(x / \bar{B}_{n}\right)$. It follows that

$$
\mathrm{P}\left(\sum_{k=1}^{n} \xi_{k} \geq C_{\eta} \bar{B}_{n} x\right) \leq \exp \left(-\eta C_{\eta} x^{2}+\eta^{2} x^{2}\right)=\exp \left(-x^{2}\right),
$$

which completes the proof.

The second and third lemmas are on the asymptotic behaviors of the largest entry of the sample covariance matrix and $\hat{\theta}_{i j}$. The proof of Lemma 2 is given in Cai and Liu (2010).

Lemma 2 (i). Under (C1), we have for any $\delta \geq 2, \varepsilon>0$ and $M>0$,

$$
\begin{gathered}
P\left(\max _{i j}\left|\hat{\sigma}_{i j}-\sigma_{i j}^{0}\right| / \hat{\theta}_{i j}^{1 / 2} \geq \delta \sqrt{\log p / n}\right)=O\left((\log p)^{-1 / 2} p^{-\delta+2}\right), \\
P\left(\max _{i j}\left\{\left|\hat{\theta}_{i j}-\theta_{i j}\right|\right\} \geq \varepsilon \sigma_{i i}^{0} \sigma_{j j}^{0}\right)=O\left(p^{-M}\right),
\end{gathered}
$$

and

$$
P\left(\max _{i}\left|\bar{X}^{i}\right| \geq C \sqrt{\log p / n}\right)=O\left(p^{-M}\right)
$$


for some $C>0$.

(ii). Under (C2), (25)-(27) still hold if we replace $O\left((\log p)^{-1 / 2} p^{-\delta+2}\right)$ and $O\left(p^{-M}\right)$ with $O\left((\log p)^{-1 / 2} p^{-\delta+2}+n^{-\epsilon / 8}\right)$ and $O\left(n^{-\epsilon / 8}\right)$ respectively.

Lemma 3 Let $\boldsymbol{X}=\left(X_{1}, \cdots, X_{p}\right)$ be a mean zero random vector. Suppose that $\operatorname{Cov}(\boldsymbol{X})=$ $I_{p \times p}$, (C3) holds and $p \rightarrow \infty$. Then under (C1) or (C2), we have for any $\delta>0$,

$$
P\left(\max _{1 \leq i<j \leq p}\left(n \theta_{i j}\right)^{-1}\left|\sum_{k=1}^{n} X_{k i} X_{k j}\right|^{2} \geq(4-\delta) \log p\right) \rightarrow 1 .
$$

Proof of Lemma 3. We arrange the two dimensional indices $\{(i, j): 1 \leq i<j \leq p\}$ in any ordering and set them as $\left\{\left(i_{m}, j_{m}\right): 1 \leq m \leq p(p-1) / 2=: L\right\}$. Let $Y_{k m}=\theta_{i j}^{-1 / 2} X_{k i_{m}} X_{k j_{m}}, \quad S_{m}=n^{-1 / 2} \sum_{k=1}^{n} Y_{k m}, \quad A_{m}=\left\{\left|S_{m}\right| \geq \sqrt{(4-\delta) \log p}\right\}, \quad 1 \leq m \leq L$.

Define $\bar{Y}_{k m}=Y_{k m} I\left\{\left|Y_{k m}\right| \leq \delta_{n} \sqrt{n /(\log p)^{3}}\right\}$ and $\hat{Y}_{k m}=\bar{Y}_{k m}-\mathrm{E} \bar{Y}_{k m}$, where $\delta_{n} \rightarrow 0$ sufficiently slow. Then by $(\mathrm{C} 1)$ or $(\mathrm{C} 2)$ we have when $n$ is large,

$$
\begin{aligned}
& \mathrm{P}\left(\max _{1 \leq i<j \leq p}\left(n \theta_{i j}\right)^{-1}\left|\sum_{k=1}^{n} X_{k i} X_{k j}\right|^{2} \geq(4-\delta) \log p\right) \\
& \geq \mathrm{P}\left(\max _{1 \leq m \leq L} n^{-1}\left|\sum_{k=1}^{n} \hat{Y}_{k m}\right|^{2} \geq(4-2 \delta) \log p\right)-O\left(p^{-M}+n^{-\epsilon / 8}\right) \\
& \geq \mathrm{P}\left(\max _{1 \leq m \leq L} n^{-1}\left|\sum_{k=1}^{n} \hat{Y}_{k m}\right|^{2} \geq 4 \log p-\log \log p+x\right)-O\left(p^{-M}+n^{-\epsilon / 8}\right) .
\end{aligned}
$$

for any $M>0$ and $x<0$. Set $y_{n}=\sqrt{4 \log p-\log \log p+x}$ and

$$
\hat{A}_{m}=\left\{n^{-1 / 2}\left|\sum_{k=1}^{n} \hat{Y}_{k m}\right| \geq y_{n}\right\} .
$$

Then by Bonferroni's inequality, we have for any fixed $l$,

$$
\mathrm{P}\left(\max _{1 \leq m \leq L} n^{-1}\left|\sum_{k=1}^{n} \hat{Y}_{k m}\right|^{2} \geq y_{n}^{2}\right) \geq \sum_{d=1}^{2 l}(-1)^{d-1} \sum_{1 \leq i_{1}<\cdots<i_{d} \leq L} \mathrm{P}\left(\bigcap_{j=1}^{d} \hat{A}_{i_{j}}\right) .
$$

Write

$$
\hat{\mathbf{Y}}_{k}=\left(\hat{Y}_{k i_{1}}, \cdots, \hat{Y}_{k i_{d}}\right), \quad 1 \leq k \leq n
$$


By Theorem 1 in Zaitsev (1987), we have

$$
\begin{aligned}
& \mathrm{P}\left(|\hat{\mathbf{N}}|_{d, \infty} \geq y_{n}-\delta_{n}^{1 / 2}(\log p)^{-1 / 2}\right)+c_{1} \exp \left(-c_{2} \delta_{n}^{-1 / 2} \log p\right) \\
& \geq \mathrm{P}\left(\left|n^{-1 / 2} \sum_{k=1}^{n} \hat{\mathbf{Y}}_{k}\right|_{d, \infty} \geq y_{n}\right) \\
& \geq \mathrm{P}\left(|\hat{\mathbf{N}}|_{d, \infty} \geq y_{n}+\delta_{n}^{1 / 2}(\log p)^{-1 / 2}\right)-c_{1} \exp \left(-c_{2} \delta_{n}^{-1 / 2} \log p\right),
\end{aligned}
$$

where $c_{1}$ and $c_{2}$ are positive constant depending only on $d,|\cdot|_{d, \infty}$ means $|\mathbf{a}|_{d, \infty}=\min _{1 \leq i \leq d}\left|a_{i}\right|$ for $\mathbf{a}=\left(a_{1}, \cdots, a_{d}\right)$, and $\hat{\mathbf{N}}$ is a $d$ dimensional normal random vector with mean zero and covariance matrix $\operatorname{Cov}\left(\hat{\mathbf{Y}}_{k}\right)$. Set

$$
\hat{B}_{i_{1}, \cdots, i_{d}}^{ \pm}=\left\{|\hat{\mathbf{N}}|_{d, \infty} \geq y_{n} \mp \delta_{n}^{1 / 2}(\log p)^{-1 / 2}\right\}
$$

We can check that $\left\|\operatorname{Cov}\left(\hat{\mathbf{N}}_{k}\right)-I_{d \times d}\right\|_{2}=O\left(1 /(\log p)^{8}\right)$. Let $\mathbf{Z}$ be a standard $d$-dimensional normal vector. Then we have

$$
\begin{aligned}
\mathrm{P}\left(\hat{B}_{i_{1}, \cdots, i_{d}}^{+}\right) \leq & \mathrm{P}\left(|\mathbf{Z}|_{d, \infty} \geq y_{n}-2 \delta_{n}^{1 / 2}(\log p)^{-1 / 2}\right) \\
& +\mathrm{P}\left(\left\|\operatorname{Cov}\left(\hat{\mathbf{N}}_{k}\right)-I_{d \times d}\right\|_{2}|\mathbf{Z}|_{2} \geq \delta_{n}^{1 / 2}(\log p)^{-1 / 2}\right) \\
= & (1+o(1))\left(\frac{1}{\sqrt{2 \pi}} p^{-2} \exp (-x / 2)\right)^{d}+O\left(\exp \left(-C(\log p)^{2}\right)\right) .
\end{aligned}
$$

Similarly we can get

$$
\mathrm{P}\left(\hat{B}_{i_{1}, \cdots, i_{d}}^{-}\right) \geq(1-o(1))\left(\frac{1}{\sqrt{2 \pi}} p^{-2} \exp (-x / 2)\right)^{d}-O\left(\exp \left(-C(\log p)^{2}\right)\right)
$$

Submitting (30)-(32) into (29), we can get

$$
\begin{aligned}
\varliminf_{n \rightarrow \infty} \mathrm{P}\left(\max _{1 \leq m \leq L} n^{-1}\left|\sum_{k=1}^{n} \hat{Y}_{k m}\right|^{2} \geq y_{n}^{2}\right) & \geq \sum_{d=1}^{2 l}(-1)^{d-1}\left(\frac{1}{\sqrt{8 \pi}} \exp (-x / 2)\right)^{d} / d ! \\
& \rightarrow 1-\exp \left(-\frac{1}{\sqrt{8 \pi}} \exp (-x / 2)\right)
\end{aligned}
$$

as $l \rightarrow \infty$. Letting $x \rightarrow-\infty$, we prove the lemma by (28) and (33). 
Proof of Theorem 1. By (C1) or (C2), we have $\theta_{i j} \leq C_{K_{1}} \sigma_{i i}^{0} \sigma_{j j}^{0}$. On the event $\left\{\max _{i j}\left|\hat{\sigma}_{i j}-\sigma_{i j}^{0}\right| \leq \lambda_{i j}\right\} \cap\left\{\hat{\theta}_{i j} \leq 2 \theta_{i j}\right.$ for all $\left.i, j\right\}$, we have by the conditions (i)-(iii) on $s_{\lambda}(z)$ that

$$
\begin{aligned}
& \sum_{j=1}^{p}\left|s_{\lambda_{i j}}\left(\hat{\sigma}_{i j}\right)-\sigma_{i j}^{0}\right| \\
= & \sum_{j=1}^{p}\left|s_{\lambda_{i j}}\left(\hat{\sigma}_{i j}\right)-\sigma_{i j}^{0}\right| I\left\{\left|\hat{\sigma}_{i j}\right| \geq \lambda_{i j}\right\}+\sum_{j=1}^{p}\left|\sigma_{i j}^{0}\right| I\left\{\left|\hat{\sigma}_{i j}\right|<\lambda_{i j}\right\} \\
\leq & 2 \sum_{j=1}^{p} \lambda_{i j} I\left\{\left|\sigma_{i j}^{0}\right| \geq \lambda_{i j}\right\}+\sum_{j=1}^{p}\left|s_{\lambda_{i j}}\left(\hat{\sigma}_{i j}\right)-\sigma_{i j}^{0}\right| I\left\{\left|\hat{\sigma}_{i j}\right| \geq \lambda_{i j},\left|\sigma_{i j}^{0}\right|<\lambda_{i j}\right\} \\
& +\sum_{j=1}^{p}\left|\sigma_{i j}^{0}\right| I\left\{\left|\sigma_{i j}^{0}\right|<2 \lambda_{i j}\right\} \\
\leq & 2 \sum_{j=1}^{p} \lambda_{i j}^{1-q}\left|\sigma_{i j}^{0}\right|^{q}+(1+c) \sum_{j=1}^{p}\left|\sigma_{i j}^{0}\right| I\left\{\left|\sigma_{i j}^{0}\right|<\lambda_{i j}\right\}+\sum_{j=1}^{p}\left|\sigma_{i j}^{0}\right| I\left\{\left|\sigma_{i j}^{0}\right|<2 \lambda_{i j}\right\} \\
\leq & C_{q, c} \sum_{j=1}^{p} \lambda_{i j}^{1-q}\left|\sigma_{i j}^{0}\right|^{q} \\
\leq & C_{K_{1}, \delta, c, q} s_{0}(p)\left(\frac{\log p}{n}\right)^{(1-q) / 2} .
\end{aligned}
$$

The proof follows from Lemma 2 and the fact $\|\boldsymbol{A}\|_{2} \leq\|\boldsymbol{A}\|_{L_{1}}$ for any symmetric matrix.

Proof of Theorems 2 and 3. Theorem 2 follows from Lemma 2 immediately. We now prove Theorem 3, For each $1 \leq i \leq p$, let $A_{1}$ be the largest subset of $\{1, \cdots, p\}$ such that $X_{i}$ is uncorrelated with $\left\{X_{k}, k \in A_{1}\right\}$. Let $i_{1}=\operatorname{argmin}\left\{|j-i|: j \in A_{1}\right\}$. Then we have $\left|i_{1}-i\right| \leq s$. Also, $\operatorname{Card}\left(A_{1}\right) \geq p-s$. Similarly, let $A_{l}$ be the largest subset of $A_{l-1}$ such that $X_{i_{l-1}}$ is uncorrelated with $\left\{X_{k}, k \in A_{l}\right\}$ and $i_{l}=\operatorname{argmin}\left\{\left|j-i_{l-1}\right|: j \in A_{l}\right\}$. We can see that $\left|i_{l}-i\right| \leq l s$ and $\operatorname{Card}\left(A_{l}\right) \geq \operatorname{Card}\left(A_{l-1}\right)-s \geq p-s l$. Take $l=\left[p^{\tau_{2}}\right]$ with $\tau^{2} / 4<\tau_{2}<\min \left(\tau^{2} / 3, \tau_{1}\right)$. Then $X_{i_{0}}, \ldots, X_{i_{l}}$ are pairwise uncorrelated random variables, where we set $i_{0}=i$. Clearly $i_{1}, \cdots, i_{l} \in B_{i}=\left\{j: \sigma_{i j}^{0}=0 ; j \neq i\right\}$. Without loss of generality, we assume that $X_{1}, \cdots, X_{l}$ are pairwise uncorrelated. Note that $\left|s_{\lambda}(z)\right| \geq|z|-\lambda$. 
It suffices to show that for some $\varepsilon_{0}>0$,

$$
\mathrm{P}\left(\max _{1 \leq i<j \leq l}\left\{\lambda_{n i j}^{-1}\left|\hat{\sigma}_{i j}\right|\right\}>1+\varepsilon_{0}\right) \rightarrow 1
$$

Clearly, we can assume $\mathrm{EX}=0$ and $\operatorname{Var}\left(X_{i}\right)=1$ for $1 \leq i \leq l$. By Lemma 2 and (14), we have $\min _{i j} \lambda_{n i j}>0$ with probability tending to one. By Lemma 2 it suffices to show that for any $0<\tau<2$,

$$
A_{n}:=\mathrm{P}\left(\max _{1 \leq i<j \leq l}\left\{\left(n \theta_{i j}\right)^{-1 / 2}\left|\sum_{k=1}^{n} X_{k i} X_{k j}\right|\right\} \geq \tau \sqrt{\log p}\right) \rightarrow 1 .
$$

Since $\tau^{2} \log p \leq(4-\delta) \log l$ for $0<\delta<4-\tau^{2} / \tau_{2}$ and large $n$, (35) follows from Lemma 3 .

Lemmas 4 and 5 below, proved in Cai and Liu (2010), are needed to prove Theorems 4 and 5 .

Lemma 4 Suppose that $\boldsymbol{X} \sim N\left(\boldsymbol{\mu}, \boldsymbol{\Sigma}_{0}\right)$ with $\boldsymbol{\Sigma}_{0} \in \overline{\mathcal{U}}_{0}$. Let $s_{0}(p)=O\left((\log p)^{\gamma}\right)$ for some $\gamma<1$ and $n^{\xi} \leq p \leq \exp \left(o\left(n^{1 / 3}\right)\right)$ for some $\xi>0$. Let $\delta>\sqrt{2}$. Then there are at most $O\left(s_{0}(p)\right)$ nonzero elements in each row of $\hat{\mathbf{\Sigma}}^{\star}(\delta)$. Furthermore,

$$
\inf _{\boldsymbol{\Sigma}_{0} \in \overline{\mathcal{U}}_{0}} P\left(\left\|\hat{\boldsymbol{\Sigma}}^{\star}(\delta)-\boldsymbol{\Sigma}_{0}\right\|_{2} \leq C_{\gamma, \delta, M} \max _{i} \sigma_{i i}^{0} s_{0}(p)\left(\frac{\log p}{n}\right)^{1 / 2}\right) \geq 1-O\left(p^{-M}\right)
$$

for any $M>0$, where $C_{\gamma, \delta, M}$ is a constant depending only on $\gamma, \delta, M$, and

$$
\sup _{\boldsymbol{\Sigma}_{0} \in \mathcal{U}_{0}} E\left\|\hat{\Sigma}^{\star}(\delta)-\boldsymbol{\Sigma}_{0}\right\|_{2}^{2} \leq C s_{0}^{2}(p) \frac{\log p}{n}
$$

for some constant $C>0$.

Lemma 5 Let $\lambda_{i j}=\tau \sqrt{\frac{\hat{\theta}_{i j} \log p}{n}}$ with $0<\tau<\sqrt{2}$. Under the conditions of Lemma 4 ,

$$
P\left(\min _{i} \sum_{j \in B_{i}} I\left\{\left|\hat{\sigma}_{i j}\right| \geq \lambda_{n i j}(\tau)\right\} \geq p^{2 \epsilon_{0}}\right) \rightarrow 1
$$

with any $\epsilon_{0}<\left(1-\tau^{2} / 2\right) / 2$, where $B_{i}=\left\{j: \sigma_{i j}^{0}=0 ; j \neq i\right\}$. Hence for some constant $C>0$

$$
\inf _{\boldsymbol{\Sigma}_{0} \in \overline{\mathcal{U}}_{0}} P\left(\left\|\hat{\boldsymbol{\Sigma}}^{\star}(\tau)-\boldsymbol{\Sigma}_{0}\right\|_{2} \geq C \min _{i} \sigma_{i i}^{0} p^{\epsilon_{0} / 2} s_{0}(p)\left(\frac{\log p}{n}\right)^{1 / 2}\right) \rightarrow 1
$$


Proof of Theorem 4. To simplify the notation, we shall write $s_{0}$ for $s_{0}(p)$. We construct a matrix $\Sigma_{0} \in \mathcal{U}_{q}^{\star}$. Let $s_{1}=\left[\left(s_{0}-1\right)^{1-q}(\log p / n)^{-q / 2}\right]+1$ and $\left(X_{1}, \cdots, X_{s_{1}}\right), X_{s_{1}+1}, \cdots, X_{p}$ be independent. Let $\sigma_{i i}^{0}=s_{0}$ for all $i>s_{1}, \sigma_{i i}^{0}=1$ for $1 \leq i \leq s_{1}$ and $\sigma_{i j}^{0}=4^{-1} s_{0} \sqrt{\log p / n}$ for $1 \leq i \neq j \leq s_{1}$. Note that $\sigma_{i j}^{0}=0$ for $i \neq j>s_{1}$. Since $s_{0}<4 \sqrt{n / \log p}, \boldsymbol{\Sigma}_{0}$ is a positive definite covariance matrix belonging to $\mathcal{U}_{q}^{\star}$. Set $\boldsymbol{M}_{n}=\left(\sigma_{i j}^{0}\right)_{1 \leq i, j \leq s_{1}}$. We first suppose that $\lambda_{n} \leq 3^{-1} \sigma_{p p}^{0} \sqrt{2 \log p / n}$. Lemma 5 yields

$$
\mathrm{P}\left(\sum_{j=s_{1}+1}^{p} I\left\{\left|\hat{\sigma}_{p j}\right| \geq \frac{\sqrt{2}}{2} \sigma_{p p}^{0} \sqrt{\frac{\log p}{n}}\right\} \geq p^{2 \epsilon_{0}}\right) \rightarrow 1,
$$

with any $\epsilon_{0}<3 / 8$. Take $\epsilon_{0}=7 / 20$ and note that $p^{1 / 4} \geq s_{0}, p^{1 / 10} \geq n^{q / 2}$. By the inequality $\left|s_{\lambda}(z)\right| \geq z-\lambda$,

$$
\inf _{\lambda_{n} \leq 3^{-1} \sigma_{p p}^{0} \sqrt{2 \log p / n}} \sup _{\mathcal{U}_{q}^{\star}} \mathrm{P}\left(\left\|\hat{\boldsymbol{\Sigma}}_{g}-\boldsymbol{\Sigma}_{0}\right\|_{2}>\frac{\sqrt{2}}{6} s_{0}^{2}(p)\left(\frac{\log p}{n}\right)^{(1-q) / 2}\right) \rightarrow 1 .
$$

We next consider the case $\lambda_{n}>3^{-1} \sigma_{p p}^{0} \sqrt{2 \log p / n}$. We have

$$
\left\|\hat{\Sigma}_{g}-\Sigma_{0}\right\|_{2} \geq\left\|\hat{M}_{n}-M_{n}\right\|_{2}
$$

where $\hat{\boldsymbol{M}}_{n}=\left(\hat{\sigma}_{i j}^{g}\right)_{1 \leq i, j \leq s_{1}}$. As in Lemma 2 , we can get for any $\gamma>0$

$$
\mathrm{P}\left(\max _{1 \leq i, j \leq s_{1}}\left|\hat{\sigma}_{i j}-\sigma_{i j}^{0}\right| \geq \sqrt{2 \gamma \log p / n}\right) \leq C s_{1}^{2}(\log p)^{-1 / 2} p^{-\gamma} .
$$

Taking $\gamma=1$, we have with probability tending to one, $\max _{1<i<j \leq s_{1}}\left|\hat{\sigma}_{i j}\right| \leq\left(4^{-1} s_{0}+\right.$ $\sqrt{2}) \sqrt{\log p / n}$, which implies that $\hat{\sigma}_{i j}^{g}=0$ for $1 \leq i \neq j \leq s_{1}$. Thus, with probability tending to one,

$$
\left\|\hat{\boldsymbol{M}}_{n}-\boldsymbol{M}_{n}\right\|_{2} \geq\left(4^{-1}-\sqrt{2} s_{0}^{-1}\right) s_{1} s_{0} \sqrt{\frac{\log p}{n}} \geq \frac{3}{64} s_{0}^{2-q}\left(\frac{\log p}{n}\right)^{(1-q) / 2} .
$$

This and (39) together imply (21).

Proof of Theorem 5 and Proposition 2, For brevity, we only consider the case $H=1$.

The proof for general $H$ is similar. We first show that for any $\varepsilon>0$,

$$
\mathrm{P}(\hat{\delta} \geq \sqrt{2}-\varepsilon) \rightarrow 1
$$


Since the random split is independent with the sample $\left\{\mathbf{X}_{1}, \cdots, \mathbf{X}_{n}\right\}$, we can assume that the two samples are $\left\{\mathbf{X}_{1}, \cdots, \mathbf{X}_{n_{1}}\right\}$ and $\left\{\mathbf{X}_{n_{1}+1}, \cdots, \mathbf{X}_{n}\right\}$. Let $\hat{\boldsymbol{\Sigma}}_{2}$ be the sample covariance matrix from $\left\{\mathbf{X}_{n_{1}+1}, \cdots, \mathbf{X}_{n}\right\}$ and $\hat{\boldsymbol{\Sigma}}_{1}^{\star}(\delta)$ be defined as in (11) from $\left\{\mathbf{X}_{1}, \cdots, \mathbf{X}_{n_{1}}\right\}$. Define

$$
\hat{\delta}_{o}=\hat{j}_{o} / N, \quad \text { where } \quad \hat{j}_{o}=\underset{0 \leq j \leq 4 N}{\arg \min }\left\|\hat{\Sigma}_{1}^{\star}(j / N)-\Sigma_{0}\right\|_{F}^{2} .
$$

Set $a_{n}=p^{-1}\left\|\hat{\boldsymbol{\Sigma}}_{1}^{\star}(\hat{\delta})-\boldsymbol{\Sigma}_{0}\right\|_{F}^{2}$ and $r_{n}=p^{-1}\left\|\hat{\boldsymbol{\Sigma}}_{1}^{\star}\left(\hat{\delta}_{o}\right)-\boldsymbol{\Sigma}_{0}\right\|_{F}^{2}$. By the proof of Theorem 1, we have $\mathrm{P}\left(\left\|\hat{\boldsymbol{\Sigma}}_{1}^{\star}(2)-\boldsymbol{\Sigma}_{0}\right\|_{L_{1}} \leq C_{1} s_{0}(p)(\log p / n)^{1 / 2}\right) \rightarrow 1$ for some $C_{1}>0$. Using the inequality $p^{-1}\|\boldsymbol{A}\|_{F}^{2} \leq|\boldsymbol{A}|_{\infty}\|\boldsymbol{A}\|_{L_{1}}$ for any $p \times p$ symmetric matrix $\boldsymbol{A}$ and the definition of $\hat{\delta}_{o}$, we have $\mathrm{P}\left(r_{n} \leq C_{2} s_{0}(p) \log p / n\right) \rightarrow 1$ for some $C_{2}>0$. Note that

$$
\mathrm{E}\left|\left(\boldsymbol{V}, \hat{\boldsymbol{\Sigma}}_{2}-\boldsymbol{\Sigma}_{0}\right)\right|^{2} \leq C n^{-1}
$$

for any $p \times 1$ vector $\boldsymbol{V}$ with $\|\boldsymbol{V}\|_{F}=1$. By the proof of Theorem 3 in Bickel and Levina (2008) and the assumption that $N$ is fixed, we can see that,

$$
a_{n} \leq O_{\mathrm{P}}\left(\frac{1}{n^{1 / 2}}\right) a_{n}^{1 / 2}+O_{\mathrm{P}}\left(\frac{1}{n^{1 / 2}}\right) r_{n}^{1 / 2}+r_{n} .
$$

Hence for some $C_{3}>0$,

$$
\mathrm{P}\left(a_{n} \leq C_{3} s_{0}(p) \log p / n\right) \rightarrow 1
$$

Note that by applying Lemma 5 to the samples $\left\{\mathbf{X}_{1}, \cdots, \mathbf{X}_{n_{1}}\right\}$,

$$
\mathrm{P}\left(a_{n} \leq C_{3} s_{0}(p) \log p / n, \hat{\delta}<\sqrt{2}-\varepsilon\right)=o(1) .
$$

This together with (42) shows that

$$
\mathrm{P}(\hat{\delta}<\sqrt{2}-\varepsilon) \leq \mathrm{P}\left(\hat{\delta}<\sqrt{2}-\varepsilon, a_{n} \leq C_{3} s_{0}(p) \log p / n\right)+o(1)=o(1),
$$

and hence (401) holds. Since $N$ is fixed, we have $|\hat{\sigma}-\sqrt{2}| \geq \varepsilon_{0}$ for some fixed $\varepsilon_{0}>0$ which depends on $N$. This together with (40) implies

$$
\mathrm{P}(\hat{\delta} \geq \sqrt{2}+\epsilon) \rightarrow 1
$$


for some $\epsilon>0$. By Lemma 4, we see that with probability tending to one, for each $i$, there are at most $O\left(s_{0}(p)\right)$ nonzero numbers of $\left\{\left|s_{\lambda_{i j}}\left(\hat{\sigma}_{i j}\right)\right| ; j \in B_{i}\right\}$ and by Lemma 2, they are of

order $O\left(\max _{i} \sigma_{i i}^{0} \sqrt{\log p / n}\right)$. Let $\Psi_{i}=\left\{j: \sigma_{i j}^{0} \neq 0\right\}$ and $\hat{\Psi}_{i}=\left\{j: \hat{\sigma}_{i j}^{\star} \neq 0\right\}$. Then by the conditions on $s_{\lambda}(z)$, we have

$$
\left\|\hat{\mathbf{\Sigma}}^{\star}(\hat{\delta})-\boldsymbol{\Sigma}_{0}\right\|_{L_{1}} \leq \max _{i} \sum_{j \in \Psi_{i} \cup \hat{\Psi}_{i}}\left|s_{\lambda_{i j}}\left(\hat{\sigma}_{i j}\right)-\sigma_{i j}^{0}\right| \leq C \max _{i} \sigma_{i i}^{0} s_{0}(p)\left(\frac{\log p}{n}\right)^{1 / 2}
$$

with probability tending to one. The proof of Theorem 5 is completed. Finally, Proposition 2 is proved by (43), Lemmas 2 and 4 .

\section{Supplemental materials}

Additional proofs: A supplement to the main paper contains additional technical arguments including the proofs of Lemmas 2, 4 and 5. (pdf file)

\section{Acknowledgment}

We thank the Associate Editor and two referees for their helpful comments which have led to an improvement of the presentation of the paper.

\section{References}

[1] Antoniadis, A. and Fan, J. (2001). Regularization of wavelet approximations. Journal of the American Statistical Association 96: 939-967.

[2] Cai, T. T. and Zhou, H. H. (2009). Minimax estimation of large covariance matrices under $l_{1}$ norm. Technical Report. 
[3] Cai, T. T. and Zhou, H. H. (2010). Optimal rates of convergence for sparse covariance matrix estimation. Technical Report.

[4] Cai, T. T. and Liu, W. (2010). Supplement to "Adaptive thresholding for sparse covariance matrix estimation". Technical Report.

[5] Bickel, P. and Levina, E. (2008). Covariance regularization by thresholding. Annals of Statistics 36: 2577-2604.

[6] Donoho, D. L. and Johnstone, J. M. (1994). Ideal spatial adaptation by wavelet shrinkage. Biometrika 81: 425-455.

[7] Donoho, D. L. and Johnstone, J. M. (1998). Minimax estimation via wavelet shrinkage. Annals of statistics 26: 879-921.

[8] Hawkins, D. L. (1989). Using $U$ statistics to derive the asymptotic distribution of Fisher's $Z$ statistic. Journal of the American Statistical Association 43: 235-237.

[9] El Karoui, N. (2008). Operator norm consistent estimation of large-dimensional sparse covariance matrices. Annals of Statistics 36: 2717-2756.

[10] Khan, J., Wei, J., Ringner, M., Saal, L., Ladanyi, M., Westermann, F., Berthold, F., Schwab, M., Antonescu, C. R., Peterson, C., and Meltzer, P. (2001). Classification and diagnostic prediction of cancers using gene expression profiling and artificial neural networks. Nature Medicine 7: 673-679.

[11] Rothman, A.J., Levina, E., and Zhu, J. (2009). Generalized thresholding of large covariance matrices. J. Amer. Statist. Assoc. 104: 177-186.

[12] Shao, Q.M. (1999). A Cramér type large deviation result for Student's t-statistic. Journal of Theoretical Probability 12: 385-398. 
[13] Wang, Y. and Zou, J. (2010). Vast volatility matrix estimation for high-frequency financial data. Annals of Statistics 38: 943-978.

[14] Zaitsev, A. Yu. (1987). Estimates of the Lévy-Prokhorov distance in the multivariate central limit theorem for random variables with finite exponential moments. Theory Probab. Appl. 31: 203-220. 